\title{
Discovery of cellular substrates of human RNA decapping enzyme Dcp2 using a stapled bicyclic peptide inhibitor
}

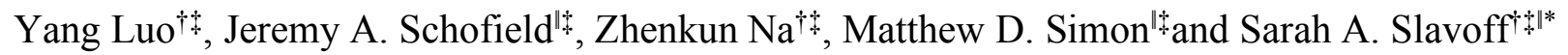 \\ 'Department of Chemistry, Yale University, New Haven, Connecticut 06520, United States \\ ${ }^{*}$ Chemical Biology Institute, Yale University, West Haven, Connecticut 06516, United States \\ 'Department of Molecular Biophysics and Biochemistry, Yale University, New Haven, Connecticut 06529, United States \\ *Correspondence: sarah.slavoff@yale.edu \\ CLASSIFICATION \\ BIOLOGICAL SCIENCES; Biochemistry
}

KEY WORDS

Dcp2; Chemical Genetics; RNA Decay; Cyclic Peptide; P-bodies

\section{AUTHOR CONTRIBUTIONS}

Y.L. designed and performed experiments and data analysis and wrote the manuscript. J.A.S and M.D.S. designed and performed TimeLapse-seq experiments and data analysis and edited the manuscript. Z.N. generated the XRN1 KO cell line and performed mass spectrometry experiments. S.A.S conceived the project, designed experiments and edited the manuscript. All authors have given approval to the final version of the manuscript.

\section{THIS PDF FILE INCLUDES:}

Main Text

Figure 1 to 6

Figure S1 to S7

Table S1

\begin{abstract}
Dcp2 is an RNA decapping enzyme that controls the stability of a subset of human RNAs including many that encode factors functioning in transcription and the immune response. While $>1800$ human Dcp2 substrates have been identified, compensatory expression changes secondary to genetic ablation of the $D C P 2$ gene have complicated a complete mapping of its regulome. Cell-permeable, selective chemical inhibitors of Dcp2 could provide a powerful tool to study Dcp2 specificity. Here, we report the selection of CP21, a bicyclic peptide ligand of human Dcp2 from chemically cyclized phage-encoded libraries. We demonstrate that CP21 has high affinity and selectivity for Dcp2 in vitro and in cells. CP21 inhibits Dcp2 decapping activity toward RNA substrates in human cells. Additionally, CP21 increases formation of P-bodies, liquid condensates enriched in intermediates of RNA decay, in a manner that resembles the deletion or mutation of Dcp2. We used CP21 to identify 76 previously undiscovered Dcp2 substrates that were subject to compensatory changes in RNA synthesis in $D C P 2$ knockout cells. This work demonstrates how CP21 and stapled peptide selection in general can provide new insights into the biology of RNA decay.
\end{abstract}

\section{SIGNIFICANCE STATEMENT}

RNA decapping, or the removal of the 7-methylguanosine $\left(\mathrm{m}^{7} \mathrm{G}\right)$ cap, is the first step that gates entry into the $5^{\prime}-3^{\prime}$ RNA decay and regulates physiologically important processes including innate immune responses. In mammals, three Nudix family proteins have been shown to function as RNA decapping enzymes, with their functional redundancy/specialization partially defined by prior studies based on traditional genetics. Genetic compensation in decapping enzyme knockout cells has been observed, complicating substrate identification for these enzymes. However, screening for inhibitors specific to each enzyme is likely challenging because of difficulties in directly targeting the similar, conformationally flexible active sites. Here, we demonstrate the selection and first application of a specific Dep2 stapled peptide ligand in the global profiling of 
Dcp2 substrates in human cells. This study demonstrates how this strategy can be used for the development of inhibitors and probes to uncover the complex network of mammalian RNA decay factors.

\section{INTRODUCTION}

Regulated RNA degradation is an important and often overlooked step in control of gene expression. While much effort has been focused on understanding synthesis and processing of RNAs, we know substantially less about the pathways that control programmed RNA decay. The first step of 5'-to-3' exonucleolytic RNA decay, one of the major pathways that regulates the stability of the cytoplasmic transcriptome in eukaryotic cells, is the removal of the 7-methylguanosine $\left(\mathrm{m}^{7} \mathrm{G}\right) \mathrm{cap}$ of deadenylated RNAs(1). In yeast this is primarily accomplished by the Nudix family decapping enzyme Dcp2(2-6). This enzyme is subject to temporal and spatial regulation by coactivator proteins including Dcp1(1), post-translational modifications(7), and phase separation into membraneless organelles known as processing bodies (P-bodies) $(8,9)$. The landscape of RNA decapping enzymes is more complex in mammals than in lower eukaryotes $(10,11)$. Mammals encode multiple Nudix family RNA decapping enzymes with distinct activities. Dcp2, Nudt16(12,13) and Nudt3(14) hydrolyze $\mathrm{m}^{7} \mathrm{G}$ caps, while Dxo/Rai1 hydrolyzes both unmethylated $(15,16)$ and NAD caps $(10,17)$. The Nudix $\mathrm{m}^{7} \mathrm{G}$ hydrolases have the potential to regulate different target transcripts despite their lack of apparent in vitro sequence specificity(13). While preliminary evidence for orthogonal regulation of genes by Dcp2, Nudt16 and Nudt3 supports this hypothesis(12, 14, 18, 19), a complete mapping of the RNA substrates of mammalian $\mathrm{m}^{7} \mathrm{G}$ decapping enzymes has not yet been achieved.

Dcp2 controls the stability of a large but specific subset of human transcripts. We recently obtained global, timeresolved measurements of RNA dynamics to identify $\sim 1800$ transcripts that are stabilized in $D C P 2$ knockout (KO) cells, suggesting that they are Dcp2 substrates(20). This and related studies have established that transcripts comprising the Dcp2 regulome are (1) enriched in P-bodies, (2) often modified with $N^{6}$-methyladenosine $\left(\mathrm{m}^{6} \mathrm{~A}\right)$, and (3) more likely to encode regulatory and immune-related factors $(13,21,22)$. We also observed a significant number of destabilized RNAs and transcriptional changes in $D C P 2 \mathrm{KO}$ cells, indicative of secondary effects as a result of genetic compensation for $D C P 2$ deletion, thereby limiting comprehensive identification of Dcp2 targets. We reasoned that the development of a specific Dcp2 inhibitor would allow acute shut down of Dcp2 activity, thereby permitting mapping of Dcp2 substrates for which changes in decay rate are masked by secondary effects in $D C P 2 \mathrm{KO}$.

Structural characterization of Dep2-containing complexes in yeast has revealed the mechanistic basis for catalysis and allosteric activation(23-28). Dcp2 uses a bipartite active site, with critical residues contributed both by the Nudix domain and the distal N-terminal regulatory domain (NRD). Yeast Dcp1/human DCP1A is a conserved activator of decapping, which contacts the Dcp2 NRD either through direct interaction with the Dcp1 EVH1 (Ena/VASP homology 1) domain in yeast(29) or through the scaffold protein EDC4 in higher eukaryotes $(30,31)$ to promote formation of a pre-catalytic closed conformation of Dcp2. Additional factors, such as yeast Edc1 $(23,26,27)$ and Edc3(28), release autoinhibition by a C-terminal Dcp2 segment and correctly position residues critical for cap recognition and hydrolysis by Dcp2 in its active conformation. It is important to note that molecular details that regulate Dcp2 activity in human may be fundamentally different from yeast, especially with regard to reprogramming of protein-protein interaction networks $(30,32)$. Given the conformational flexibility and coactivator requirements of Dcp2, directly targeting the catalytic site with small-molecule inhibitors is likely to be challenging(33). Furthermore, Dcp2 has low affinity for most small molecule cap analogs, requiring an intact, capped RNA substrate of at least 12 residues(34), complicating in vitro screening.

Despite these challenges, Dcp2 inhibitors have been reported. These bivalent cap analogues, consisting of two $\mathrm{m}^{7} \mathrm{G}$ moieties joined by a 5'-to-5' tetraphosphate bridge to increase binding affinity, can bind to and inhibit the active conformation of Dcp2 at the catalytic site $(25,33)$. The D3 diastereomer of the most potent analog, bearing $\alpha$ - and $\delta$-phosphorothiolate substitution, exhibited a $K_{\mathrm{I}}$ of $121 \pm 16 \mu \mathrm{M}$ for Schizosaccharomyces pombe Dcp2 (spDcp2)(33). These inhibitors exhibit increased affinity toward the reconstituted spDcp $1 / 2$ complex compared to spDcp2 alone, suggesting that they bind to the active conformation and making then particularly valuable for structural and mechanistic studies $(25,26)$. These dinucleotide cap analogues are membrane impermeable, however, and also have limited affinity and, likely, specificity for Dcp2, making them poorly suited for use in determining the RNA substrates of Dcp2 in cells.

To develop new inhibitors of Dcp2, one major challenge is the absence of a preformed, high affinity substrate binding pocket in Dcp2 for ligand or inhibitor binding. To address this challenge, we employed a strategy that could target surface or allosteric sites using proteomimetics(35). Chemically stapled or cyclized peptides are emergent classes of ligands developed to target large protein surfaces inaccessible by small molecules(36-38). In addition to mimicking structured protein-binding domains, these molecules feature resistance to proteolytic degradation compared to their linear equivalents in vivo, and can display detectable membrane permeability $(39,40)$. We were particularly inspired by work from Heinis et al., which reported a phage-encoded stapled bicyclic peptide library that enables in vitro selection of ligands for protein targets(41). Here, we applied this approach to identify a cyclic peptide, CP21, which functions as a selective and cell-permeable human Dcp2 ligand. We demonstrate that CP21 increases the stability of Dcp2 target RNAs and P-body formation, consistent with inhibition, and use this molecule to identify 76 Dcp2 substrates invisible to previous studies based on genetic ablation of $D C P 2(13,20)$. Together, our data illustrates the utility of selected cyclic peptides in the biochemical characterization and substrate identification of human Dcp2, establishing this approach as an attractive platform to study the biology of RNA decay enzymes. 


\section{RESULTS}

Phage display screening identifies CP21 as a ligand of human Dcp2

We hypothesized that chemically stapled bicyclic peptides might achieve specificity for binding Dcp2 through interactions with one or more large surfaces on the protein, as previously reported for human urokinase-type plasminogen activator (uPA)(42). We used an initial peptide library that consisted of two fully randomized 6-mer peptide sequences flanked by a total of three cysteine residues displayed on a disulfide-free phage pIII protein. The phage displayed peptides were chemically cyclized with 1,3,5-tris(bromomethyl)benzene (TBMB), as previously described(43) (Figure 1a). We immobilized Dcp2 with a site-specific biotinylation strategy(44) to avoid blocking important surfaces of the enzyme, as would occur using less directed chemistry. Specifically, we appended a 15-amino acid BirA biotin ligase substrate, AviTag peptide(45), to the Cterminus of full-length Dcp2 (Dcp2 $2^{\mathrm{FL}}-\mathrm{AP}$ ), and the tagged protein was labeled in vitro using purified BirA and biotin. Successful biotinylation was confirmed using streptavidin enrichment (Figure S1a-c). Biotinylated Dcp2 ${ }^{\mathrm{FL}}$-AP was immobilized on streptavidin beads for phage display selection. Starting with $10^{10}$ purified TBMB-modified phage particles, three rounds of selection were performed, including amplification of enriched phage and chemical cyclization of the displayed sequences before each round of selection. To facilitate enrichment of high-affinity Dcp2 binders, the stringency of selection was increased in the $2^{\text {nd }}$ and the $3^{\text {rd }}$ round by doubling the number of wash steps and decreasing the amount of immobilized Dcp2 $2^{\mathrm{FL}}$-AP. A beads-only negative control was included in all steps to identify non-specific streptavidin binders. One hundred colonies were sequenced from the last round of selection, and we identified two classes of sequences (Figure 1b): the first class was enriched in positively charged amino acids and proline; the second class, which we did not pursue farther given the high likelihood that these members displayed peptides prone to aggregation and/or nonspecific binding, was characterized by highly hydrophobic and aromatic residues. Further considering sequence consensus in the first class (Figure 1b, top), we observed that proline was strongly preferred in position 1 of the first loop, and enriched in position 1 of the second loop as well as positions 5 or 6 of both loops. Enrichment of positively charged amino acids or glutamine was observed in position 3 ( $\mathrm{H}, \mathrm{K}$ or Q) and position $4(\mathrm{H})$ of loop 1. Weaker convergence was observed in loop 2. New affinity maturation libraries were then constructed to recapture the convergence in the first class by fixing proline in the first and either the fifth or the sixth position of loop 1, together with a histidine or a glutamine at the fourth position of loop 1. The remaining positions were randomized. These libraries were mixed and panned using stringent conditions (40 to $10 \mathrm{pmol}$ of Dcp $2^{\mathrm{FL}}-\mathrm{AP}$ ). Sequencing clones from the enriched library revealed emergence of a new, albeit weak, consensus with preference for proline at position 3 of loop 2 flanked by positively charged residues (Figure 1c). Representative sequences from this selection were chosen for further characterization.

\section{CP21 interacts with Dep2 in vitro}

We were encouraged that the affinity maturation revealed a new Dcp2 binding consensus. To identify an optimal Dcp2 ligand from the enriched members, we screened individual members using an orthogonal in vitro binding assay. The DNA sequences of enriched peptides from both primary selection and affinity maturation were expressed, purified and TBMBcyclized as peptide-D1D2-FLAG (disulfide-free phage protein pIII domains) fusions to test their interaction with GST-Dcp2 ${ }^{\mathrm{FL}}$ (Figure S1b,d). The top hit, CP21 (Figure 2a), arose from affinity maturation, and its D1D2 fusion showed 15-fold higher enrichment of Dcp2 compared to the control (unfused D1D2 phage protein) in this assay (Figure 2b). We then chemically synthesized and cyclized CP21 containing the same core $6 \times 6$ sequence with an extra N-terminal alanine and C-terminal amidated glycine appended(41). This synthetic peptide construct was utilized for all subsequent experiments and hereafter is indicated by the term CP21 (Figure 2a and Figure S2a). The $K_{\mathrm{D}}$ of CP21 for the catalytically active Dcp2 ${ }^{1-349}$ truncation construct(46) (Figure S1a,b) was $116 \pm 31 \mathrm{nM}$ by isothermal titration calorimetry (ITC) (Figure 2c), while its affinity for another cytoplasmic decapping enzyme, full-length human Nudt16, was approximately 300-fold lower $\left(K_{\mathrm{D}}=34.5 \pm 1 \mu \mathrm{M}\right)$ (Figure 2d). Additionally, the synthetic all-D enantiomeric form of CP21 (D-CP21, Figure S2b) showed undetectable binding to Dcp2 $2^{1-349}$ in ITC (Figure 2e), demonstrating that the 3-dimensional orientation of the sidechains, rather than the general biophysical properties of the peptide, was responsible for binding. These results support our conclusion that CP21 binds specifically to Dcp2.

CP21 binding requires a C-terminal disordered segment of Dcp2

To map the Dcp2 interface responsible for CP21 binding, we used photo-cross-linking-based affinity enrichment and proteomics. To make a CP21 analogue with a photo-crosslinker that retains binding, we replaced residues that were not conserved in the most enriched peptide sequences from the phage display selection with photoreactive amino acids, L-PhotoMethionine (Photo-M) or L-Photo-Leucine (Photo-L), at either loops or near the cyclization center (Photo-CP-1 to -3, Figure S2c-e and Figure S3a). Biotin was added to the N-terminus of all peptides through an alkyl linker as an affinity handle for enrichment of crosslinked proteins. Reaction with Photo-CP-3 led to the greatest enrichment of purified Dcp2 ${ }^{1-349}$ over background (Figure S3b). We then performed mass spectrometry analysis of pre- and post-Photo-CP-3-cross-linked Dep2 $2^{1-349}$ (Figure S3c). Three candidate sites of interaction were found at or near the Nudix domain, and one more was found near the C-terminal FEB (phenylalanine-rich EDC4 binding) motif known to interact with EDC4, an important scaffolding protein of the active human decapping complex $(30,32)$. This unexpected C-terminal candidate led us to more closely examine the functional relevance of binding to the disordered C-terminal region of Dcp2. 
We hypothesized that the disordered C-terminal region may be involved in Dcp2 activity or protein interactions, which may be disrupted by CP21 binding. Recent structural studies of yeast Dcp2 demonstrated the functional importance of intrinsically disordered regions in regulatory autoinhibition(28), but the C-terminus of human Dcp2 is distinct in sequence from that of yeast and has not been characterized beyond the FEB motif. We therefore examined the interaction of CP21 with the Dep2 C-terminus. We generated alanine mutants of GST-tagged Dcp $2^{\mathrm{FL}}$ between amino acids 325 and 333 and examined binding with CP21-D1D2-FLAG containing the candidate interaction site for CP21 (Figure S3c, blue). Q332A or K333A mutations had the largest effect on binding, leading to $\sim 50 \%$ reduction in the amount of CP21 fusion enriched from the pulldown compared to wild-type Dcp2 (Figure S3d). We hypothesized that CP21 could remodel protein-protein interactions within the decapping complex by binding to the C-terminus of Dcp2. Intriguingly, we found a depletion of DDX6 from the decapping complex immunoprecipitated from CP21 vs D-CP21 treated HEK293T cells (Figure S4). However, DDX6 does not directly contact Dcp2 and was shown instead to interact with the human decapping machinery via EDC3(47). It is hence possible that CP21 affects recruitment of DDX6 and/or its bound RNAs indirectly. Together, these data suggest that CP21 can affect decapping complex composition by engaging with a disordered C-terminal region of Dep2.

CP21 specifically binds to Dcp2 in cells

To investigate the ability of CP21 to selectively engage Dcp2 in the presence of other cellular proteins, we used PhotoCP-3 to examine direct binding of CP21 to Dcp2 in whole cell lysates (Figure 3a). We incubated Photo-CP-3 with HEK293T cell lysates ectopically expressing Dcp2 and compared enrichment in the presence or absence of 5-fold excess of unlabeled competitor CP21 $\left(\left[\mathrm{CP} 21_{\text {unlabled }}\right]=300 \mu \mathrm{M}\right)$. Quantitative proteomics revealed enrichment of Dcp2 over all other proteins, supporting Dcp2 as the primary cellular target of CP21 (Figure 3b).

Cyclized peptides can be relatively cell permeable compared to their linear counterparts $(39,48)$. We therefore asked whether the CP21 peptide could penetrate the cell membrane. N-terminally FITC-labeled CP21 with an aminohexanoic (Ahx) acid spacer was chemically synthesized, purified (Figure S2f, g) and its cellular uptake examined by live-cell fluorescence confocal microscopy. HEK293T cells incubated with FITC-Ahx-CP21 or the all-D enantiomer $(3 \mu \mathrm{M}, 5 \mathrm{~h})$ exhibited comparable internalization (Figure 3c). While a majority of observed fluorescence was punctate, consistent with endosomal localization(40), the presence of a low level of diffuse cytoplasmic signal indicated detectable endosomal escape.

To evaluate Dcp2-CP21 interaction in the cytoplasm, a cellular thermal shift assay (CETSA) was performed in HEK293T cells(49). In comparison to the vehicle-treated group, the melting temperature of Dcp2 was increased by $3.2^{\circ} \mathrm{C}$ when cells were treated with $2 \mu \mathrm{M} \mathrm{CP} 21$, while no significant difference was observed when cells were treated with D-CP21 (Figure 3d). Together with selective cross-linking of Photo-CP-3 to Dcp2 in lysates, these results suggest CP21 preferentially binds to Dcp2 in cells.

CP21 inhibits Dep2 in cells

We next investigated the specificity and functional consequence of CP21 toward Dep2 activity inside cells by measuring the transcript levels of known Dcp2 substrates RNAs using qRT-PCR. Previously, Kiledjian and coworkers reported RRP41 RNA as a preferred substrate of human Dcp2(19). More recently, we demonstrated that HOXA13 RNA is a Dcp2 target (20). We thus used these two transcripts to evaluate the effect of cellular CP21 delivery on Dcp2 activity. Briefly, cells were pre-treated with compounds, then transcription was inhibited with actinomycin D and RNA levels assessed at specified timepoints to measure decay. Both Dep2 substrate RNAs were significantly stabilized by CP21 $(2 \mu \mathrm{M})$ compared to controls (vehicle or D-CP21) (Figure 4a). The K333A mutant of Dcp2 (which abrogates CP21 binding) also led to stabilization of $R R P 41$ when this mutant was expressed on a DCP2 KO background in HEK293T cells (Figure S5a-b), demonstrating that this mutation phenocopies CP21 treatment and supporting functional importance of the Dcp2 C-terminus. The fact that D-CP21 did not alter the stability of all tested RNAs indicates that the observed inhibition of RNA decay was specific to inhibitory activity of CP21 in its all-L form. Importantly, we found that CP21 did not lead to a further increase in the stability of the RRP41 transcript in the $D C P 2 \mathrm{KO}$ cell line, suggesting that CP21 mediates its RRP41-stabilizing effect specifically through Dcp2 and not via off-target inhibition or other non-specific effects (Figure 4b). Stability of a non-Dcp2 substrate RNA, PLBD2(20), remained unchanged by CP21 treatment (Figure 4c), further supporting a specific effect on Dcp2-mediated decay pathways.

To more directly measure RNA decapping in cells, we performed the splinted ligation assay to quantify decapped RNA, which accumulates to detectable concentrations in yeast or mammalian cells lacking the downstream exonuclease Xrn1 $(20,50)$. We measured the decapping of the HOXA13 RNA in XRN1 KO cells (20) treated with $2 \mu \mathrm{M}$ CP21, D-CP21 or vehicle (Figure 4d). The transient accumulation of decapped HOXA13 RNAs (the product of Dcp2 activity) was specifically abolished in CP21-treated cells. These results demonstrate that CP21 stabilizes RNAs by inhibiting either the decapping activity of Dcp2, or its interaction with RNA substrates, in cells.

CP21 inhibition of Dep2 might be expected to phenocopy knockout, silencing or inactivating mutation of Dcp2. Pbodies are cytoplasmic liquid domains enriched in translationally repressed RNAs in complex with RNA decay-associated proteins $(8,51)$. Deletion or silencing of Dcp2 has been shown to increase P-body numbers in yeast $(52)$ and mammalian cells(53), possibly due to accumulation of deadenylated RNA decay intermediates in complex with proteins that can phase separate at high local concentrations. We observed that expression of the Dcp2 K333A mutant (in DCP2 KO cells) caused a modest but statistically significant 1.3-fold increase in P-body numbers relative to expression of wild-type Dcp2, consistent with Dcp2 inhibition (Figure S5c). We thus asked whether CP21 treatment would also affect P-body numbers. Indeed, CP21 
increased P-body numbers compared to vehicle-treated cells with an effect size similar to the K333A mutant (Figure 4e; average 1.4-fold increase), while P-body numbers in D-CP21 treated cells remained unaltered. Taken together, our RNA stability and P-body imaging data suggest that CP21 inhibits the cellular function of Dcp2.

CP21 enables identification of new Dep2 substrates

The above data support our conclusion that CP21 inhibits Dcp2-dependent turnover of endogenous reporter RNAs in cells. To explore the utility of CP21 in mapping of RNA substrates of Dcp2, we assessed the impact of CP21 on RNA stability using TimeLapse-seq in wild-type (WT) and DCP2 KO HEK293T cells pre-treated with D-CP21 or CP21 ( $2 \mu \mathrm{M}, 5 \mathrm{~h})$ using previously reported conditions (20). This shortened treatment time ( $5 \mathrm{~h}$ compared to $18 \mathrm{~h}$ for previous experiments) was chosen to minimize compensatory responses to Dep2 inhibition and led to partial inhibition of Dcp2-mediated decay of a known RNA substrate (RRP41, Figure S6). We assigned 104 RNAs as specifically stabilized by CP21 using previously established criteria (20), among which the majority (91 transcripts) showed smaller relative change of decay rate $\left(\frac{\Delta k_{d e g}}{\Delta k_{s y n}}\right)$ in $D C P 2 \mathrm{KO}$ vs WT HEK293T cells and were therefore defined as Dcp2 substrates (Figure 5a and Table S1). The majority of these RNAs (84\%) were missing from our previous report on Dcp2 substrates using a cutoff of significant changes at total RNA level(20). We hypothesized that these substrates were previously undetected as a result of secondary, compensatory effects of $D C P 2$ genetic ablation that obscured variation at total RNA level. We selected several CP21-stabilized transcripts identified in this study, and reanalyzed previously reported changes in their decay and synthesis rates in DCP2 KO vs WT HEK293T cells(20). Indeed, $58 \%$ exhibited a decreased decay rate in the $D C P 2 \mathrm{KO}$ relative to WT, among which $68 \%$ evidenced a simultaneous decrease in RNA synthesis and therefore no significant change in total RNA level in DCP2 KO cells. This trend is evident in TimeLapseseq sequencing tracks of these RNAs in $D C P 2 \mathrm{KO}$ vs WT cells (Figure S7).

We validated several new Dcp2 substrates revealed by CP21 inhibition using an independent assay based on transcriptional arrest and qRT-PCR. First, we confirmed that RNA stabilization is specific to CP21 treatment as compared to treatment with the D-CP21 control for six RNAs that were stabilized in the TimeLapse-seq dataset, either included in $(R F X 7$, $A R H G A P 32, P A X 6)$ or missing from (SRSF12, ANKRD13A, ERGIC2) our previous Dcp2 substrates list (Figure 6a and Figure S7). To corroborate the observed upregulation as depending on Dcp2 inhibition, half-lives of the same RNAs in DCP2 KO cells were then compared to those in WT HEK293T cells after transcriptional arrest (Figure 6b). RNA stability was greater in the $D C P 2 \mathrm{KO}$ cells relative to WT HEK293T for all tested transcripts, and the magnitude of stabilization of each transcript in $D C P 2 \mathrm{KO}$ cells was comparable to stabilization observed upon $\mathrm{CP} 21$ treatment.

\section{DISCUSSION}

In this work, we developed a cell-permeable bicyclic peptide inhibitor of Dcp2 activity in human cells. Importantly, this inhibitor (CP21) selectively enriches Dcp2 over other cellular proteins, including related RNA binding proteins and decapping enzymes. The specificity of CP21 for Dcp2 binding and modulation is further supported by the inactivity of its enantiomer, D-CP21, which is otherwise identical in amino acid composition and charge. In addition to directly binding the Nudix domain of Dcp2, we also found evidence that CP21 binds a C-terminal disordered region of Dcp2. We demonstrate the importance of this region in human Dcp2 by showing that Dcp2 target decay rates are slowed and P-bodies are increased by the K333A Dcp2 mutation. CP21 may exert its cellular effect on Dcp2 activity by decreasing binding of DDX6, likely indirectly, preventing RNA substrate delivery. This work highlights the need to further study the role of C-terminus of human Dcp2, and its role in human Dcp2 activation. Addressing this important question will be facilitated by CP21.

A significant increase in cellular P-body numbers was observed upon CP21 treatment, phenocopying the effects of perturbed RNA decay described in prior reports(52) and that we observed with a K333A Dcp2 mutant (Figure S5c). Further supporting the dependence of this effect on Dcp2 binding, D-CP21 had no detectable effect on P-bodies. The increase of Pbody numbers by CP21 may be a result of the local accumulation of deadenylated RNA that cannot be decapped by Dcp2, leading to phase separation, consistent with Dcp2 inhibition by CP21.

Global profiling of RNA levels using TimeLapse-seq in WT and DCP2 KO HEK293T cells pre-treated with CP21 revealed Dcp2-dependent stabilization of 91 RNAs that were not stabilized in the D-CP21 control, with 15 of these RNAs, mostly transcription factors, being previously described as Dcp2 substrates(20). Inspection of the 76 novel Dcp2 substrates identified here reveal splicing and transcription factors as well as proteins involved in signaling transduction (e.g. GTPases), among other important functions. 52\% of CP21-stablized RNAs were upregulated by DDX6 silencing in a previous study(54), consistent with altered DDX6 association with the decapping complex by CP21. Importantly, we provide evidence that many Dcp2 substrates identified in this study were previously masked by increased synthesis rates in $D C P 2 \mathrm{KO}$ cells, demonstrating the value of acute inhibition in overcoming genetic compensation.

While this study successfully identified a number of novel Dep2 targets, several important questions remain. First, only 91 transcripts were stabilized by CP21 — far fewer than the 1800 Dcp2 substrates previously identified(20). This is most likely due to incomplete inhibition of Dcp2 by CP21, first and foremost because of the short pretreatment used in the TimeLapse-seq experiments (Figure S6). It is also likely that CP21 is not fully membrane permeable, leading to low effective concentration of the inhibitor within the cytosol. In vitro $\mathrm{IC}_{50}$ measurements would be necessary to determine if Dcp2 is fully inhibited at the experimental CP21 concentration and treatment times. Second, the Dcp2 substrates identified in this study, as a class, diverge in their properties from Dcp2 substrates identified in DCP2 KO. For example, $60 \%$ of Dcp2 targets identified 
in this work are depleted from P-bodies relative to the cytoplasm - close to the proportion of RNAs in the whole transcriptome that are depleted from P-bodies(55) - in contrast to our previous study of Dcp2 substrates, a majority of which were enriched in P-bodies(20). This suggests that CP21 may inhibit activity toward a subset of Dcp2 substrates distinct from those stabilized by genetic knockout. It is possible that some Dcp2 substrates are selectively sensitive to Dcp2 inhibition by CP21; in a study of yeast Dep2 active site vs C-terminal mutants, differential stabilization of RNA substrates by different mutations was observed, providing precedent for this hypothesis(56).

In summary, this study reports a selective Dcp2 ligand that can be used to inhibit the decay of specific Dcp2 substrates and increase membraneless organelle formation. Application of CP21 in global profiling of Dcp2 substrates reiterates the complexity of human 5'-3' RNA decay and extends our knowledge of Dcp2 substrate specificity. Future efforts that focus on further biochemical and structural characterization of the Dcp2-CP21 interaction; determination of the effect of CP21 on Dep2 structure, interactions, and activity in cells; and systematic evaluation of the cellular uptake of CP21 is likely to provide new insights into the molecular and conformational control of Dep2 activity.

\section{MATERIALS AND METHODS}

Cell Culture. HEK293T cells were purchased from ATCC, and early-passage stocks were maintained to ensure cell line provenance and sterility. DCP2 KO and XRN1 KO HEK293T cells were generated using gRNAs to target the genomic region, as described earlier(20). All cells were maintained in DMEM (Invitrogen) supplemented with 10\% (vol/vol) FBS and $100 \mathrm{U} / \mathrm{mL}$ penicillin-streptomycin. Cells were verified to be mycoplasma-free using the ATCC Universal mycoplasma detection kit.

Antibodies. Primary antibodies used for western blotting and/or immunofluorescence were as follows: mouse monoclonal anti-FLAG (Sigma, F3165); rabbit polyclonal anti-Dcp2 (Novus Biologicals, NBP1-41070); mouse monoclonal anti-beta actin (Invitrogen, BA3R); rabbit polyclonal anti-DCP1A (Sigma, D5444); rabbit polyclonal anti-GST-tag (GenScript, A00202); rabbit polyclonal anti-DDX6 (abcam, ab103308); and streptavidin horseradish peroxidase conjugate (ThermoFisher, S911). Secondary antibodies for western blotting were goat anti-rabbit peroxidase conjugate (Rockland, 611-103-122; Merck, AP132P) and goat anti-mouse peroxidase conjugate (Rockland, 6101319). The secondary antibody for immunofluorescence was goat anti-rabbit Alexa Fluor 568 (Life Technologies, A-11011).

Generation of phage peptide libraries. The phage-encoded 6 × 6 peptide library was cloned into the fd0D12 vector (kindly provided by Dr. Christian Heinis and Dr. Simon Middendorp from the Institute of Chemical Sciences and Engineering in EPFL, Lausanne) with SfiI restriction sites. The cysteine-free D1D2 domain of phage pIII was amplified from fdg3p0ss21, and genes encoding the semi-random peptide sequence were appended to its N-terminus in two consecutive PCR reactions, as previously described by Heinis and co-workers(43). The desalted ligation product was transformed into electrocompetent TG1 cells (Lucigen) and plated on 2-YT/chloramphenicol (30 $\mu \mathrm{g} / \mathrm{mL})$ agar plates. Colonies were scraped off from the plates with 2-YT media, supplemented with $15 \%$ glycerol and stored at $-80{ }^{\circ} \mathrm{C}$. The phage library was purified from 1-liter TG1 culture inoculated with the glycerol stock by polyethylene glycol (PEG) precipitation, and the infective virions were determined by plating 10 -fold dilutions on 2-YT/chloramphenicol plates. The isolated phage was incubated with $1 \mathrm{mM}$ tris(2carboxyethyl) phosphine (TCEP) in $20 \mathrm{~mL}$ of degassed reaction buffer $\left(5 \mathrm{mM}\right.$ EDTA, $\left.20 \mathrm{mM} \mathrm{NH}_{4} \mathrm{HCO}_{3}, \mathrm{pH} 8.0\right)$ for $1 \mathrm{~h}$ at $42{ }^{\circ} \mathrm{C}$ to reduce all cysteines, followed by cyclization with $12.5 \mu \mathrm{M}$ tris(bromomethyl)benzene (TBMB) in reaction buffer/acetonitrile (vol/vol=4:1) at $42{ }^{\circ} \mathrm{C}$ for $1 \mathrm{~h}$ and a final PEG precipitation step. A thiol quantification assay (Abcam) was utilized to confirm successful cyclization. Phage titer at all stages of phage production and cyclization was analyzed to ascertain that no significant loss of infective virions was observed.

Cloning and genetic constructs. His 6 -BirA was obtained from Addgene as a gift from A. Ting(57) (Stanford University, Department of Genetics \& Biology, Stanford, CA). For bacterial expression, full-length Dep2 (Dcp2 ${ }^{\mathrm{FL}}$ ) was cloned into BamHI and SalI sites of the pGEX6P-3 vector (GE Life Sciences) together with a C-terminal His 6 tag or into SalI and XhoI sites of the pET21a(+) vector with a C-terminal AviTag peptide (AP)(44) (Supplementary Figure 1b). All truncations of Dcp2 were cloned into BamHI and XhoI sites of pET28a for affinity purification by His 6 tag (Supplementary Figure 1b). Selected Dcp2-binding peptide sequences were appended to the N-terminus of the cysteine-free phage D1D2 domain of pIII with a C-terminal FLAG tag by PCR and cloned into pET28a between NcoI and HindIII sites, adding an additional C-terminal His 6 motif (“peptide-D1D2"). Alanine mutants of GST-Dcp2 ${ }^{\mathrm{FL}}$ were generated using inverse PCR.

Recombinant protein production. All His 6 -tagged Dcp2 truncations and Dcp2 $2^{\mathrm{FL}}$-AP were purified by TALON resin (Clontech) as previously reported $(18,58)$. Briefly, Dcp2 expression was induced in BL21 Gold (DE3) cells with $0.4 \mathrm{mM}$ isopropyl $\beta$-d-thiogalactoside (IPTG) at $20^{\circ} \mathrm{C}$ overnight. Bacterial pellets were lysed by sonication in ice-cold lysis buffer A $\left(50 \mathrm{mM} \mathrm{Na}_{2} \mathrm{HPO}_{4}, \mathrm{pH} \mathrm{7.5,600} \mathrm{mM} \mathrm{NaCl}\right.$, $300 \mathrm{mM}$ urea, $10 \%$ glycerol, $1 \%$ Triton X-100) supplemented with $1 \mathrm{mg} / \mathrm{mL}$ lysozyme and DNaseI. The lysate was incubated with preequilibrated TALON beads at $4{ }^{\circ} \mathrm{C}$ for $1 \mathrm{~h}$, and the resin was washed first with wash buffer $\mathrm{A}\left(50 \mathrm{mM} \mathrm{Na}_{2} \mathrm{HPO}, \mathrm{pH} 7.5,600 \mathrm{mM} \mathrm{NaCl}, 10\right.$ $\mathrm{mM}$ imidazole, $300 \mathrm{mM}$ urea, $0.5 \%$ Triton X-100) followed by wash buffer $\mathrm{A}_{0}$ without Triton X-100 and urea. Bound protein was eluted with elution buffer ( $50 \mathrm{mM} \mathrm{Na}_{2} \mathrm{HPO}_{4}, \mathrm{pH} 7.5,600 \mathrm{mM} \mathrm{NaCl}, 300 \mathrm{mM}$ imidazole). Fractions containing Dep2 were combined, concentrated and dialyzed against storage buffer $(20 \mathrm{mM}$ Tris, $\mathrm{pH} 7.2,100 \mathrm{mM} \mathrm{NaCl}, 1 \mathrm{mM}$ DTT, $1 \mathrm{mM} \mathrm{MgCl} 2,10 \%$ glycerol) for downstream applications. His 6 -BirA was expressed and purified as previously reported(44).

Phage selections with Dcp2 $2^{\mathrm{FL}}$-AP. Three rounds of bio-panning were implemented with purified Dcp2 $2^{\mathrm{FL}}-\mathrm{AP}(2-5 \mu \mathrm{g})$ that was biotinylated by recombinant BirA(44) and immobilized on streptavidin magnetic beads (NEB, S1420) as described previously(43). In brief, isolated phage library and $50 \mu \mathrm{L}$ streptavidin beads with or without immobilized Dcp $2^{\mathrm{FL}}-\mathrm{AP}$ were separately blocked with blocking buffer containing $3 \%(\mathrm{w} / \mathrm{v}) \mathrm{BSA}$ and $0.3 \%$ Tween-20 and mixed for $30 \mathrm{~min}$ at room temperature. The beads were washed 8 times with $1 \mathrm{~mL}$ wash buffer (10 $\mathrm{mM}$ Tris-HCl, $\mathrm{pH} 7.4,150 \mathrm{mM} \mathrm{NaCl}, 10 \mathrm{mM} \mathrm{MgCl}, 1 \mathrm{mM} \mathrm{CaCl}_{2}, 0.1 \%$ Tween-20) and twice with $1 \mathrm{~mL}$ buffer without Tween-20 before elution with $100 \mu \mathrm{L}$ of $50 \mathrm{mM}$ glycine, $\mathrm{pH}$ 2.2. The beads were transferred to different tubes every three washes to minimize non-specific phage binding to tubes. Eluted phages were neutralized with $50 \mu \mathrm{L}$ of $1 \mathrm{M}$ Tris- $\mathrm{HCl}, \mathrm{pH} 8.0$ and incubated with $25 \mathrm{~mL}$ of TG1 cells at OD 600 of 0.4 for $90 \mathrm{~min}$ at $37^{\circ} \mathrm{C}$ without shaking. The cells were spun down, suspended in $1 \mathrm{~mL}$ of 2-YT and plated on large 2-YT/chloramphenicol plates. Plasmid DNA of $>100$ phage clones from the third round was sequenced (Genewiz, South Plainfield, NJ). 
GST pull-down assay. Wild-type and mutant GST-Dep2 $2^{\mathrm{FL}}$ were expressed in Rosetta cells (DE3) in LB medium with $0.4 \mathrm{mM}$ IPTG at 20 ${ }^{\circ} \mathrm{C}$ overnight. After cell lysis by sonication in lysis buffer A $\left(50 \mathrm{mM} \mathrm{Na} \mathrm{HPO}_{4}, \mathrm{pH} 7.5,600 \mathrm{mM} \mathrm{NaCl}, 300 \mathrm{mM}\right.$ urea, $10 \%$ glycerol, $1 \%$ Triton X-100) supplemented with lysozyme, DNase I and protease inhibitor cocktail (Roche), proteins were affinity purified sequentially using TALON matrix (Clontech) and glutathione agarose beads (GE Healthcare) according to manufacturer's instruction. All peptide-D1D2FLAG-His 6 fusion proteins were expressed in BL21 Gold (DE3) cells with $1 \mathrm{mM}$ IPTG at $20^{\circ} \mathrm{C}$ overnight and lysed in lysis buffer B (30 $\mathrm{mM} \mathrm{Na} \mathrm{HPO}_{4}, \mathrm{pH} 7.4,300 \mathrm{mM} \mathrm{NaCl}, 1 \mathrm{mM}$ TCEP, $0.1 \%$ Triton X-100) supplemented with lysozyme, DNase I and protease inhibitor cocktail. All peptide-D1D2 fusions were purified on TALON resin and cyclized with TBMB as previously described(43). GST-pull-downs using these purified proteins were then carried out in PBS by incubating mixtures of $0.4 \mu \mathrm{M}$ GST-Dcp2 $2^{\mathrm{FL}}$ and $4 \mu \mathrm{M}$ cyclic peptide-D1D2FLAG-His $_{6}$ on $25 \mu \mathrm{L}$ glutathione beads at $4{ }^{\circ} \mathrm{C}$ overnight. The beads were washed twice with 5 bed volumes of PBS, $0.5 \%$ Tween 20 followed by four PBS washes. Bound proteins were eluted with $30 \mu \mathrm{L}$ of $2 x$ sample buffer and analyzed by SDS-PAGE followed by Western blotting.

Chemical synthesis of bicyclic peptides. All linear peptides were synthesized and derivatized at the C-terminus with amide and, for live cell imaging of peptide uptake, at the N-terminus with FITC through an Ahx linker by solid-phase chemistry (GenScript). All photo-crosslinkable linear peptides with N-term biotin were ordered from New England Peptide. For chemical cyclization, the crude linear peptides $(0.5$ $\mathrm{mM}$ ) dissolved in degassed $80 \%$ (v/v) $20 \mathrm{mM} \mathrm{NH}_{4} \mathrm{HCO}_{3}, 5 \mathrm{mM}$ EDTA, pH 8.0 and 20\% MeCN were each reacted with $1 \mathrm{mM}$ TBMB at 30 ${ }^{\circ} \mathrm{C}$ for $90 \mathrm{~min}$. The reaction product was purified by reverse phase HPLC using a semi-prep C18 column (Agilent) and gradient elution with a mobile phase composed of $\mathrm{MeCN}$ and $0.1 \%(\mathrm{v} / \mathrm{v})$ aqueous formic acid solution at a flow rate of $1 \mathrm{~mL} \mathrm{~min}^{-1}$. The purified cyclic peptides (CP) were lyophilized and re-dissolved in degassed peptide storage buffer (50 mM Tris- $\mathrm{HCl}, \mathrm{pH} 7.8,150 \mathrm{mM} \mathrm{NaCl})$ for downstream applications. Product was assessed by liquid chromatography-mass spectrometry (Agilent 6550A QTOF), with mass spectra obtained by electrospray in positive ion mode(59). Quantitation was achieved from the HPLC absorbance peak area (220 nm) for CP21 in all-L or all-D form using LP25 as the standard and by UV absorbance at $495 \mathrm{~nm}$ for FITC-labeled CP21 with an extinction coefficient of $77000 \mathrm{M}^{-1} \mathrm{~cm}^{-1}$ (39).

Isothermal Calorimetry (ITC). Nano ITC (TA Instruments) was used to carry out the ITC analysis. All peptides used for the assay were lyophilized and re-dissolved in the same buffer containing $20 \mathrm{mM}$ Tris- $\mathrm{HCl}(\mathrm{pH} 7.2), 100 \mathrm{mM} \mathrm{NaCl}$, and $1 \mathrm{mM} \mathrm{MgCl}_{2}$. The titrations were carried out at $293 \mathrm{~K}$, with $100 \mu \mathrm{M}$ of Dep $2^{1-349}$ in the sample cell as titrate and $1 \mathrm{mM}$ of CP2 1 in either isoform in the syringe as titrant. Data analysis was carried out using Nano Analyze provided by Nano ITC. All ITC data were fitted with a one-site binding model with baseline subtracted.

In vitro photo-cross-linking. Photo-cross-linking was performed in a microtiter plate on ice with purified His ${ }_{6}$-Dcp $2^{1-349}(1 \mu \mathrm{M})$ and each photo-CP $(10 \mu \mathrm{M})$ in DPBS. The reaction mixture was pre-incubated at room temperature for 30 min prior to UV irradiation at $365 \mathrm{~nm}$ for 15 min. Samples were analyzed by SDS-PAGE followed by western blotting with an antibody to the Dcp2 or with Strep-HRP.

Immunoprecipitation of RNA decapping complex. HEK293T cells stably expressing FLAG-tagged DCP1A in $10 \mathrm{~cm}$ dishes were treated with either $2 \mu \mathrm{M}$ CP2 1 or D-CP21 at 70\% confluency for $18 \mathrm{~h}$. Cells were then harvested and lysed on ice using $1 \mathrm{~mL}$ TBS with $1 \%$ Triton X-100 supplemented with Roche Complete protease inhibitor cocktail (Sigma Aldrich). 10\% cell lysate were saved for analysis of input. A $50 \mu \mathrm{L}$ aliquot of pre-equilibrated anti-FLAG agarose beads (clone M2, Sigma Aldrich) was added to each cell lysate and incubated at $4{ }^{\circ} \mathrm{C}$ for $1 \mathrm{~h}$. The beads were washed 4 times with TBS-T and eluted with one bed volume of $3 \times$ FLAG peptide (Sigma Aldrich) at a final concentration of $1 \mathrm{mg} / \mathrm{mL}$ in TBS. Beads were removed by centrifugation, and eluates were analyzed by SDS-PAGE followed by western blotting or subjected to on-bead trypsin digestion followed by Mass spectra analysis.

Photo-cross-linking in cell lysates and proteomics (Target ID). HEK293T cells were transiently transfected with FLAG-Dcp2 ${ }^{\mathrm{FL}}$-pcDNA3. After $24 \mathrm{~h}$ of transfection, cells were harvested and lysed in radioimmunoprecipitation assay (RIPA) buffer supplemented with protease inhibitors (Roche). $300 \mu \mathrm{L}$ of whole cell lysate diluted to $1 \mathrm{mg} / \mathrm{mL}$ in DPBS was added to each reaction mixture. Photo-CP-3 was then added at $50 \mu \mathrm{M}$ concentration, with or without $250 \mu \mathrm{M} \mathrm{CP} 21$ as competitor. Reaction mixtures were incubated at $4{ }^{\circ} \mathrm{C}$ with rotating for $1 \mathrm{~h}$, followed by UV crosslinking $(365 \mathrm{~nm}, 23 \mathrm{~W})$ on ice for $1 \mathrm{~h} .10 \mu \mathrm{L}$ samples were taken and subjected to SDS-PAGE followed by western blotting analysis. The remaining samples were supplemented with $3 \%$ BSA and loaded on $60 \mu \mathrm{L}$ streptavidin magnetic beads (NEB) pre-washed with RIPA buffer, followed by $1 \mathrm{~h}$ incubation at room temperature. The beads were washed twice with $1 \mathrm{~mL}$ RIPA buffer, once with $1 \mathrm{M}$ $\mathrm{KCl}$, once with $0.1 \mathrm{M}$ sodium carbonate, once with $2 \mathrm{M}$ urea, and twice with RIPA buffer. On-bead trypsin digestion and ultramicrospin clean-up were performed before run on a Thermo Q-Exactive Plus instrument. Mass spectra were analyzed using Mascot against uniprot_human database, and label-free quantitation (LFQ) was carried out using MaxQuant(60) followed by Perseus(61).

Cellular thermal shift assay (CETSA). HEK293T cells in $100 \mathrm{~mm}$ dishes were treated with either $2 \mu \mathrm{M}$ CP2 1 or its all-D form (D-CP21) or the same volume of vehicle (peptide storage buffer, $50 \mathrm{mM}$ Tris- $\mathrm{HCl}, \mathrm{pH} 7.8,150 \mathrm{mM} \mathrm{NaCl}$ ) at $70 \%$ confluency. After $18 \mathrm{~h}$, cells were harvested, washed twice with DPBS and resuspended in $1 \mathrm{~mL}$ PBS containing one tablet of protease inhibitors (Roche). Each cell suspension was distributed into ten PCR tubes and heated to temperatures ranging from 45 to $65{ }^{\circ} \mathrm{C}$ for 3 min using an MJ Research PTC-200 PCR thermal cycler, followed by incubation at room temperature for $3 \mathrm{~min}$. The samples were freeze-thawed twice using liquid nitrogen, vortexed briefly and spun down at $20,000 \mathrm{~g}$ for $20 \mathrm{~min}$ at $4{ }^{\circ} \mathrm{C}$. The supernatant was then removed and analyzed by SDS-PAGE followed by western blotting(49).

Generation of stable rescue cell lines. $D C P 2 \mathrm{KO}$ HEK293T cells were co-transfected with wild-type or alanine mutant Dcp $2^{\mathrm{FL}}$ cloned into $\mathrm{pLJM} 1$, along with pMD2.G and psPAX2. Media containing viruses was harvested $48 \mathrm{~h}$ after transfection, aliquoted and keep at $-80{ }^{\circ} \mathrm{C}$ prior to transduction. Stable cell lines were generated by transducing with diluted virus stock (1:3) at around $70 \%$ cell confluency for $48 \mathrm{~h}$. Cells were harvested $48 \mathrm{~h}$ after puromycin selection, and expression level of Dcp2 was validated by western blotting.

Cell-based RNA decay assay. DCP2 KO or wild-type (WT) HEK293T cells were cultured in 6-well plates and treated with either $2 \mu \mathrm{M}$ $\mathrm{CP} 21$ or D-CP21 or the same volume of vehicle at $50 \sim 70 \%$ confluency for $18 \mathrm{~h}$. Alternatively, respective cells were cultured to a confluency of $80 \sim 90 \%$ before experiments. Cells were harvested on ice at the indicated time points after transcriptional arrest by actinomycin D (5 $\mu \mathrm{g} / \mathrm{mL}$ ). Total RNA was isolated with RNeasy Mini kit (Qiagen) or TRIzol (Invitrogen) following the manufacturer's instructions, followed 
by DNase I (NEB) treatment. RNA levels were quantified using reverse-transcribed cDNA from the same amount of input RNAs by realtime PCR using iTaq Universal SYBR Green Supermix (Bio-Rad) based on a comparative Ct method as described previously(50). In brief, RNA amounts remaining at each time point relative to time $=0 \mathrm{~h}$ were calculated using $2^{\Delta \mathrm{Ct}}$, where $\Delta \mathrm{Ct}=\mathrm{Ct}(\mathrm{t}=0)-\mathrm{Ct}($ time point $)(50)$. Alternatively, relative RNA level was normalized to $\beta$-actin before subjected to analysis by comparative Ct method(62). All data points were then fit into an exponential decay model. Two-tailed $t$-test or analysis of variance (ANOVA) was performed using Excel, GraphPad Prism 7 or Origin 2018. Equal variance between samples was established using an $F$-test, and multiple comparisons were performed with Dunnett's test following one-way ANOVA. Previously reported primers were used for RRP41(19) and HOXA13(20). Forward and reverse primers used for other genes were as follows (Gene-Fwd/-Rev): PLBD2-Fwd: 5' GGCCCCTTCGAGTATGAAGTC 3'; PLBD2-Rev: 5' TCCTGCATCCACTCTAGGTTG 3'; RFX7-Fwd: $\quad$ 5' $^{\prime}$ ACTGGAGATGGGTTGGAAGG 3'; RFX7-Rev: 5' AGACGGCAAGCAGAAGAGAT 3'; ARHGAP32-Fwd: $\quad$ 5' CCATGCCTGGAGGTTTAGTT 3'; ARHGAP32-Rev: 5'

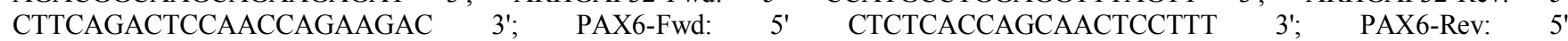
CCCTTTATGAGGCATCCTTTCT 3'; SRSF12-Fwd: 5' AGAAATAGGAGGCGGTCAGA 3'; SRSF12-Rev: 5' TTGACTGCCTTGCTGAGGTA $\quad$ 3'; ANKRD13A-Fwd: $\quad$ 5' TGCACCTCCTAGTCTGGAAAA 3'; ANKRD13A-Rev: 5' AGATGCAATAATGTTCGACCTCG $\quad$ 3'; ERGIC2-Fwd: 5' GGACCATAACACAAGCATGACTA 3'; ERGIC2-Rev: 5' TCTCTACATAGCTCTCAGGAACC 3'.

P-body imaging using confocal microscopy. HEK293T cells were grown on fibronectin-coated glass coverslips in a 48-well plate to 50\% confluency and treated with $2 \mu \mathrm{M} \mathrm{CP} 21$ or D-CP21 or the same volume of vehicle for $18 \mathrm{~h}$. Cells were fixed with $10 \%$ neutral buffered formalin (Fisher Scientific), permeabilized with methanol at $-20^{\circ} \mathrm{C}$, and blocked with blocking buffer ( $3 \%$ BSA in DPBS) for $1 \mathrm{~h}$ at room temperature. Cells were stained with rabbit anti-DCP1A(63) at a 1:1000 dilution in blocking buffer overnight at $4{ }^{\circ} \mathrm{C}$, followed by $3 \mathrm{x}$ PBS washes. Goat anti-rabbit Alexa Fluor 568 was applied at a 1:1000 dilution in blocking buffer for 1 to $4 \mathrm{~h}$ at room temperature in dark, with 3 subsequent PBS washes. Cells were post-fixed with 10\% buffered formalin, stained with DAPI, and imaged by laser scanning confocal microscopy (Leica TCS SP8) with PL APO 63x/1.40 oil, CS2 and PL APO 100x/1.44 oil, CORR CS. P-body numbers were counted using Image J as described previously(64).

Live cell imaging. HEK293T cells were grown on glass coverslips in a 48-well plate to $70 \%$ confluency and treated with $3 \mu \mathrm{M}$ FITC-AhxCP21, FITC-labeled all-D CP21 (FITC-Ahx-D-CP21) or the same volume of vehicle for $5 \mathrm{~h}$. The growth medium was aspirated immediately before imaging. The cells were washed three times with pre-warmed PBS and covered in PBS for immediate room-temperature analysis by confocal microscopy with HCX PL APO 100x/1.44 oil objective (Leica TCS SP8).

TimeLapse-seq. TimeLapse-seq experiment was performed as previously described. Briefly, wild-type (WT) HEK293T and DCP2 KO cells were pre-treated with $2 \mu \mathrm{M} \mathrm{CP} 21$ or D-CP2 $1(5 \mathrm{~h})$ in duplicate followed by incubation with $500 \mu \mathrm{M} \mathrm{s} \mathrm{U}$ for $2 \mathrm{~h}$, and a single replicate control per cell line was not treated with $\mathrm{s} \cdot \mathrm{U}$. Total RNA was isolated from cell pellets using TRIzol reagent, followed by phenol-chloroform extraction and isopropanol precipitation supplemented with $50 \mu \mathrm{M}$ DTT. After washing twice with $75 \%$ ethanol, dried RNA pellet was resuspended in nuclease-free water and treated with Turbo DNase to remove genomic DNA. RNA was isolated using RNAclean beads, treated with TimeLapse chemistry (2,2,2-trifluoroethylamine and sodium periodate) and purified as previously described(65). Purified recoded RNA was then sequenced using the mammalian pico-input SMARTer stranded Total RNA-seq kit v2. Paired-end $100 \mathrm{nt}$ or $150 \mathrm{nt}$ RNA sequencing was performed using an Illumina Hiseq-4000 or Novaseq. For data analysis, TimeLapse-seq reads were trimmed and aligned to mature RNA isoforms, and mutation calling was performed as described previously(65). Assignment of new and old sequencing reads using per-read mutation cutoffs was performed as described in Luo et al. 2020 (20). Old and new read counts per gene were normalized per sample based on total RNA counts using EdgeR. Log (fold change) analysis was performed using DEseq2 on total, new, and old RNA counts, with scale factors from total RNA input into new and old RNA analyses. Change in RNA synthesis and degradation rates were determined using the following equations: $k_{\mathrm{dg}}=-\log (1-$ $\left.f_{\mathrm{new}}\right) / \mathrm{t} ; k_{\mathrm{sp}}=[\mathrm{RNA}] * k_{\mathrm{dsg}}$, where $f_{\mathrm{new}}$ is the fraction of new RNA and [RNA] is the normalized total RNA read count per gene.

Quantitative splinted ligation RT-PCR (qSL-RT-PCR). The detection of decapped HOXA13 RNA by qSL-RT-PCR was performed in XRN1 KO HEK293T cells as previously described(20). In brief, XRN1 KO cells were cultured in 6-well plates and subjected to specified treatment (CP21, D-CP21, or vehicle) for $18 \mathrm{~h}$. Actinomycin D $(5 \mu \mathrm{g} / \mathrm{mL})$ was added to stop transcription, and cells were harvested on ice at the indicated time points after transcriptional arrest. Total RNA was isolated with the RNeasy Mini kit, and $10 \mu \mathrm{g}$ total RNA was mixed with 10 pmol of each splint oligonucleotide and 30 pmol of anchor RNA (5'GCUGAUGGCGAUGAAUGAACACUGCGUUUGCUGGCUUUGAUG-3'). The oligos were annealed by sequential incubation for 5 min from $70{ }^{\circ} \mathrm{C}$ to $60{ }^{\circ} \mathrm{C}$ to $42{ }^{\circ} \mathrm{C}$ and to $25^{\circ} \mathrm{C}$, followed by the ligation step with $2 \mu \mathrm{L}$ T4 DNA ligase (NEB) at $16^{\circ} \mathrm{C}$ overnight. $20 \mathrm{U}$ RNasin Plus (Promega) was included to prevent RNA degradation. $2 \mu \mathrm{L}$ DNase I (NEB) was then added to digest genomic DNA for $1 \mathrm{~h}$ at $37{ }^{\circ} \mathrm{C}$. The RNA was precipitated with $0.3 \mathrm{M}$ sodium acetate ( $\mathrm{pH}$ 5.2) and 2.5 volume of ethanol at $-20^{\circ} \mathrm{C}$ overnight, washed with $70 \%$ ethanol and resuspended in $15 \mu \mathrm{L}$ of DEPC-treated water. cDNA was synthesized from $2 \mu \mathrm{g}$ of the ligated RNA with iScript kit (Bio-rad). Total RNA and splinted ligation product level was then quantified by real-time PCR using gene specific Fwd/Rev primers and Anchor Fwd/Rev primers(20). A $\Delta \Delta \mathrm{Ct}$ method was used to determine the relative level of decapped RNA (50), and the amounts of total RNA remaining at each time point relative to time $=0 \mathrm{~h}$ were calculated from $2^{\Delta \mathrm{Ct}}$ using $\mathrm{Ct}(\mathrm{t}=0 \mathrm{~h})$ as the reference value. Analysis of variance (ANOVA) was performed using Excel, GraphPad Prism 7 or Origin 2018. Equal variance between samples was established using an $F$-test, and multiple comparisons were performed with Dunnett's test following one-way ANOVA.

Data availability. All TimeLapse-seq data were submitted to GEO (accession code GSE153258; full data can be accessed using this link with the following reviewer's token crolkeyepxuhdwf: https://www.ncbi.nlm.nih.gov/geo/query/acc.cgi?acc=GSE153258).

\section{ACKNOWLEDGMENT}

We thank Dr. Christian Heinis (Institute of Chemical Sciences and Engineering, Ecole Polytechnique Fédérale de Lausanne, Lausanne, $\mathrm{CH}$ ) for providing phage vectors (fdg3p0ss21 and fd0D12) and expert advice. We thank all members of the Slavoff lab for helpful 
discussion and comments on the manuscript. We also thank Prof. J. Ellman, A. Saghatelian, and J. Crawford for valuable suggestions on the project. This work was supported in part by the NIH (R01GM122984), the Searle Scholars Program, a Catalyst Award from the Dr. Ralph and Marian Falk Medical Research Trust, and Yale University West Campus start-up funds (to S.A.S.) and by NIH Grant DP2 HD083992-01 (to M.D.S). J. A. S. was in part supported by an NIH Predoctoral Training Grant (T32GM007223).

\section{COMPETING INTERESTS}

The authors declare no conflict of interests. 
a

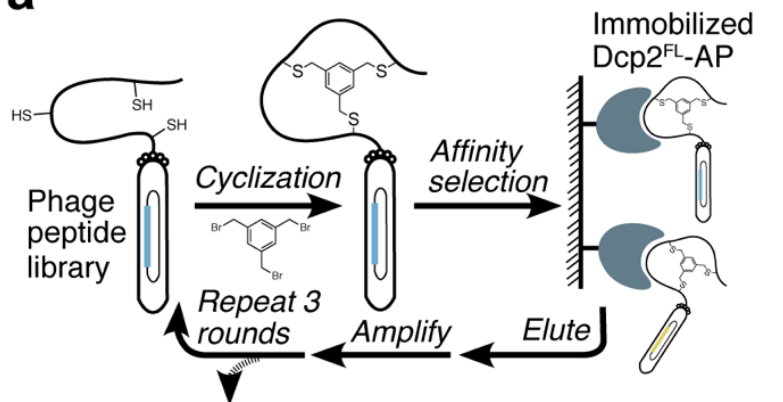

DNA sequencing, SPPS, and binding

C and decapping assays

Library 5:

P A M A A C P X X H X P C X X X X X G G S A E

Mutant: Amino acid sequence:

CP16

CP17

CP18

CP19

CP20

CP21

CP22

CP23

CP24

CP25

CP26

CP27$$
\text { A C P L L H K P C P N R Q L P }
$$
A C P V Q H E P C P N L N L P C A C P V K H D C H I N I P N C A C P D T H A P C S I A R A C A C P A T H K P C H Q P L P Q C A C P K T H P P C S K P K T Q C A C P K T H P P C N K P K T $Q$ A C P K N H N P C S A T Q P E A C P Q N H S P C H Y G I F C A C P N R H S P C R L V E V C A C P S M H P C Q I N T L P C A C P G P H P C Q I V I D P C
Abundance b

$\begin{gathered}\text { Random } \\ \text { loop } 1\end{gathered}$
$\begin{gathered}\text { Cys1 } \\ \text { Cys2 }\end{gathered}$

Mutant: Amino acid sequence: Abundance

CP1 A C P L Q Q P K C L S K T S A C 1

CP2 AC P Q Q E P S C K Q L L T I C 1

CP3 ACEKK S A G M E D Q V C

CP4 A C N H K P H C N Y E Q V C 1

CP5 A C H V T Q P C S Q T T P C 1

CP6 ACD S K H Q M T Q Q Y K T

CP7 A C S N H N K P C P H H A P H C

CP8 ACPTKHRPCPNW T T C 1

$\begin{array}{llll}\text { CP9 A C P H H R C K A L E P S C } & 1\end{array}$

$\begin{array}{lllllll}\text { CP10 A P L } Q \text { H S P C H R H S P C } & 1\end{array}$

CP11 ACRV E H W P Q T P T R

CP12 ACFFLRV L L L W GFWC 4

CP13 ACFLCRLVCLFVFF C 2

CP14 AC GF Y L F C FIF F F F C

CP15 ACVVILSFCFFESFC

Positively charged Proline Aromatic

Figure 1. Phage display selection of bicyclic peptides to identify ligands of human Dcp2 (Dcp2). (a) Schematic illustration of the strategy to select Dcp2 binders from a phage-displayed 6x6 peptide library containing 3 fixed cysteine residues for chemical cyclization with 1,3,5-tris(bromomethyl)benzene (TBMB). (b) Sequences of two classes of selected bicyclic peptides enriched by a site-specifically biotinylated Dcp2 construct, Dcp2 ${ }^{\mathrm{FL}}$-AP (see Figure S1). Positively charged residues are highlighted in red, prolines orange, and aromatic residues blue. (c) Amino acid sequences of selected clones from affinity maturation with Dcp $2^{\mathrm{FL}}-\mathrm{AP}$. All clones derive from library 5 , designed around the positively charged and proline-rich sequences enriched from the initial fully random library (b, top). 
a

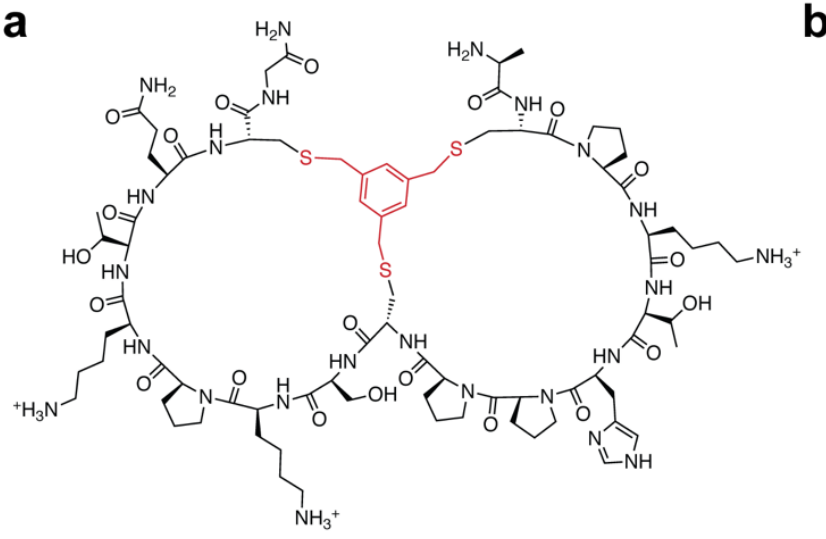

C

CP21 vs Dcp2 $2^{1-349}$

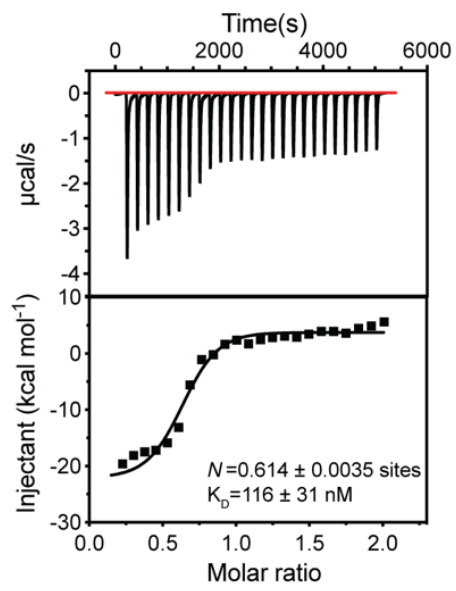

d b

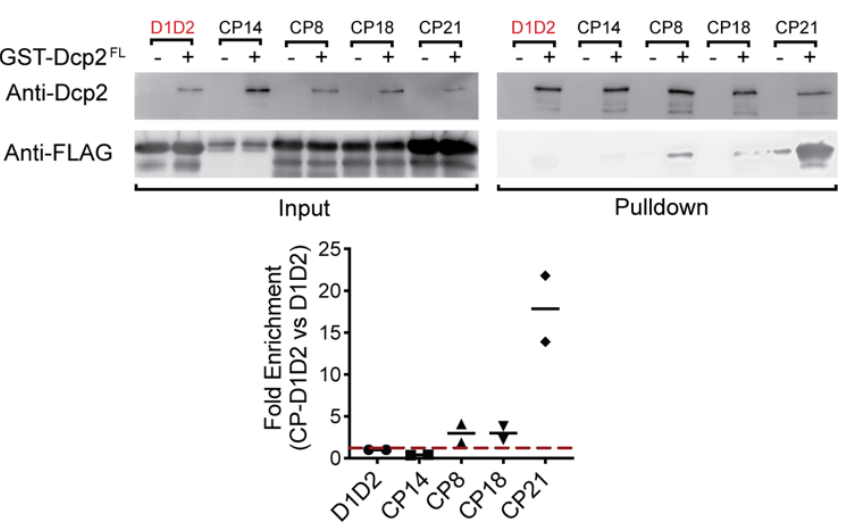

CP21 vs Nudt16

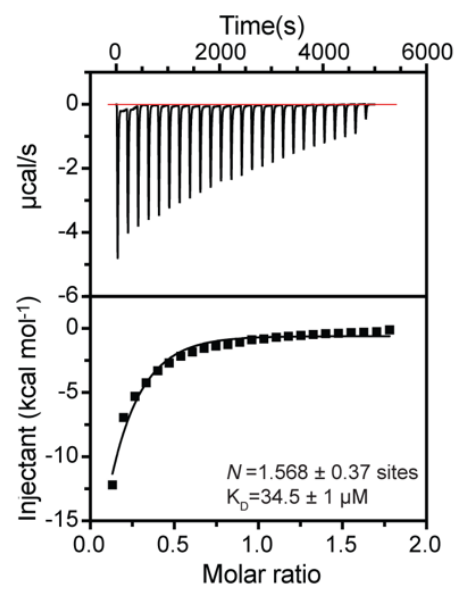

e

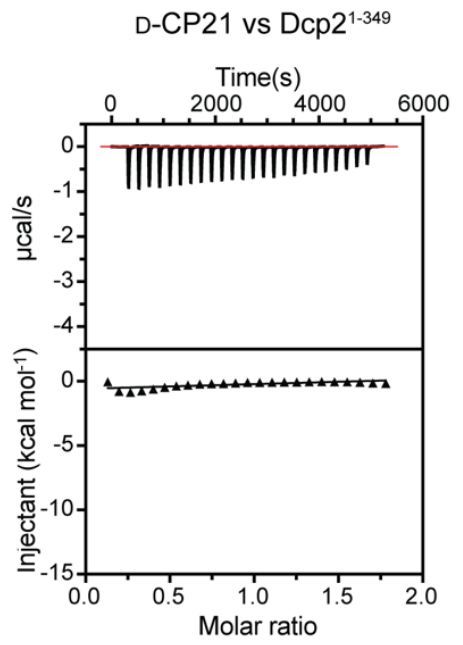

Figure 2. Cyclic peptide CP21 binds to Dep2 in vitro. (a) Chemical structure of CP21. (b) Validation of Dep2 binding to selected TBMB-cyclized peptides by in vitro pull-down assay. Phage-display-enriched CP sequences were selected and cloned as fusions to the cysteine-free D1D2 domain of phage pIII protein for bacterial expression, purified and chemically cyclized. GST-Dcp $2^{\mathrm{FL}}$ was coincubated with 10-fold molar excess of the FLAG epitope-tagged CP-D1D2 fusion proteins, with D1D2 alone as a negative control, and GST pull-down was performed prior to western blot detection. Representative data of two technical replicates are shown. Lines from the scatter plot indicate the mean value of CP-D1D2 enrichment. (c-e) Isothermal calorimetry (ITC) titrations of chemically synthesized CP21 to Dep2 ${ }^{1-349}(\mathrm{c})$, CP21 to Nudt16 (d), and D-CP21 to Dep2 ${ }^{1-349}$ (e), respectively. Data shown are representative of two technical replicates, and dissociation constant is reported as \pm the error of fitting. 
a

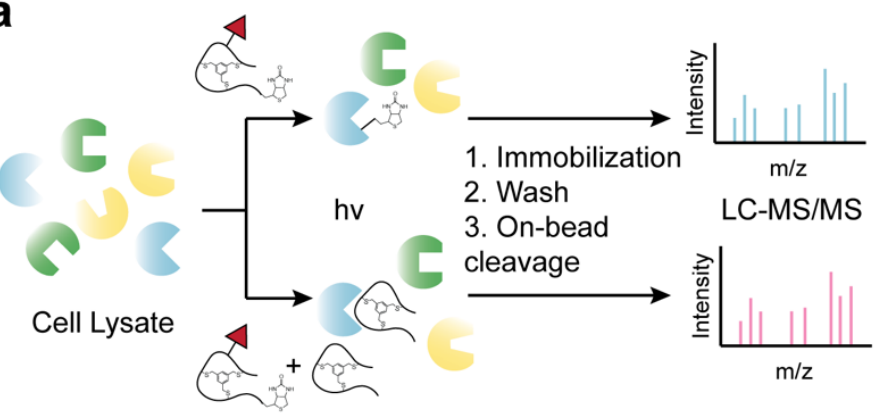

C

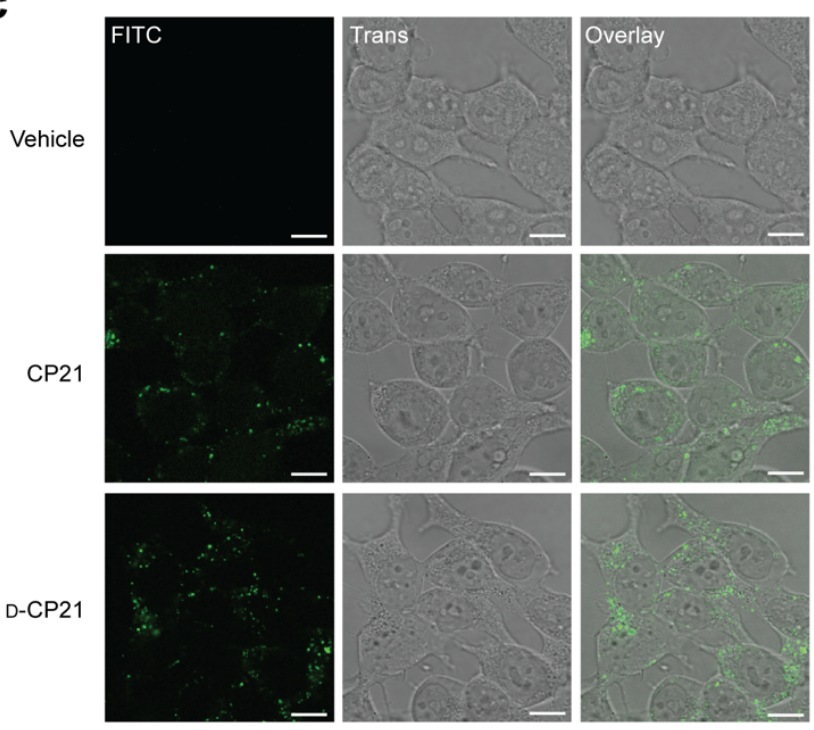

b

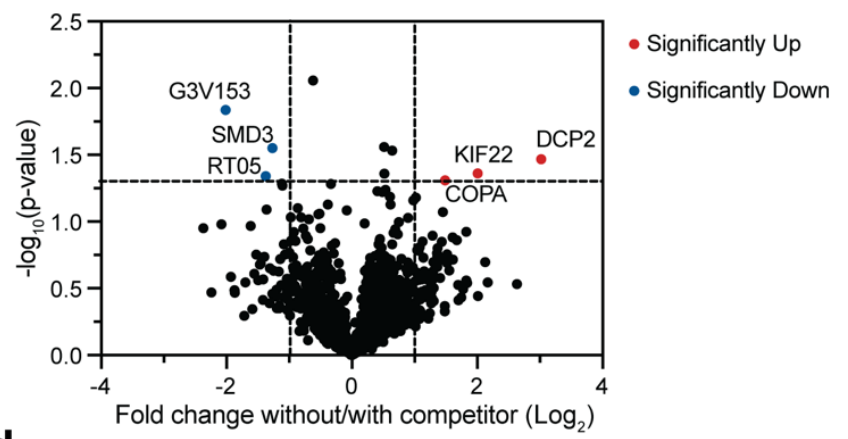

d

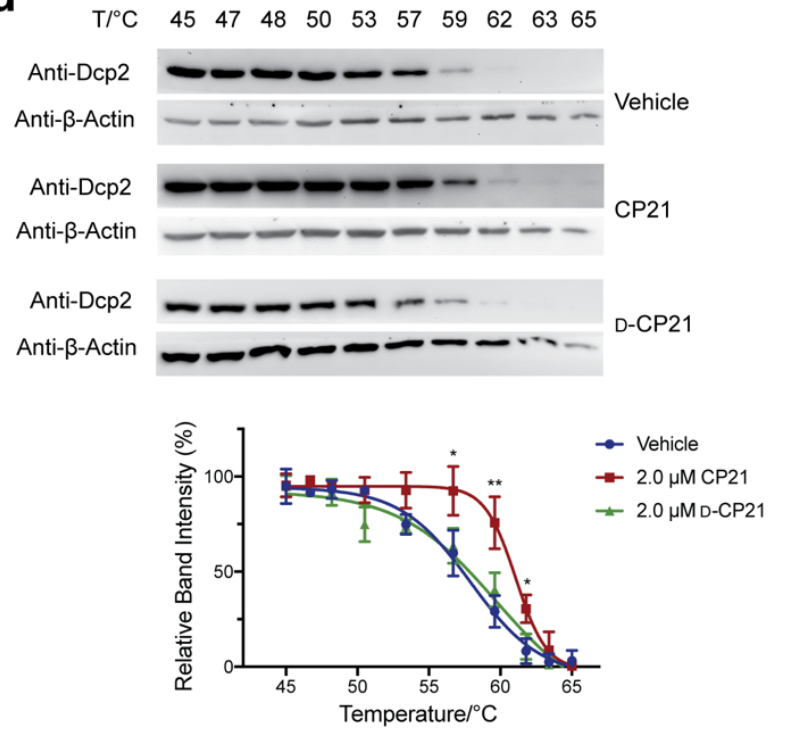

Figure 3. CP21 selectively binds to Dep2 in cells. (a) Schematic representation of the target ID workflow. (b) Volcano plot of protein enrichment ratios (without/with 5-fold excess of unlabeled CP21 as competitor) and p-values from target ID experiments $(n=3)$. (c) Assessment of cellular uptake of the cyclic peptides by live-cell confocal fluorescence microscopy of HEK293T cells incubated with vehicle or FITC-labeled CP21 in either all-L or all-D form $(3 \mu \mathrm{M}, 5 \mathrm{~h})$. Z-plane scanning was performed to minimize signal from cell surface. Images are representative of two independent experiments. Scale bars, $10 \mu \mathrm{m}$. Trans, transillumination. (d) Cellular thermal shift assay (CETSA) of Dcp2 in the presence and absence of $2 \mu \mathrm{M} \mathrm{CP} 21$ in intact HEK293T cells. D-CP21 was used as a negative control. Significant differences in Dcp2 intensity (band densitometry) at 57,59 and $62{ }^{\circ} \mathrm{C}$ were analyzed by one-way ANOVA. Number of biological repeats: $n=3$. Error bars represent \pm s.d. P-values (with Dunnett's test) are denoted by asterisks; $\left({ }^{*}\right) \mathrm{p}<0.05 ;\left({ }^{* *}\right) \mathrm{p}<0.01$. 
a

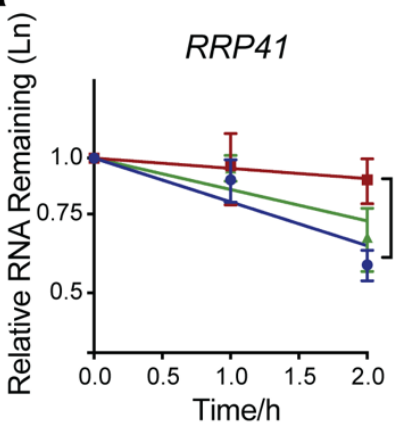

C

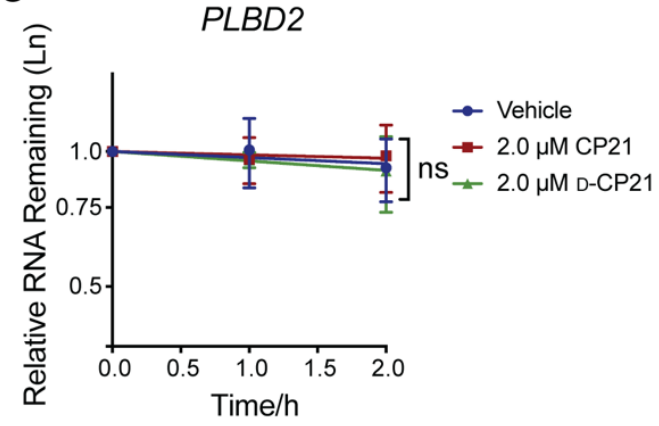

$\mathbf{e}$

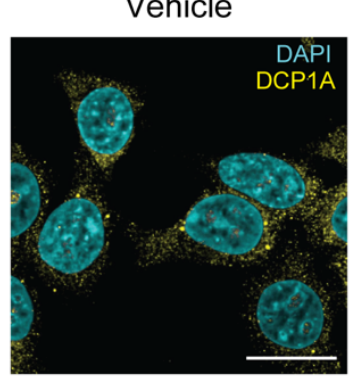

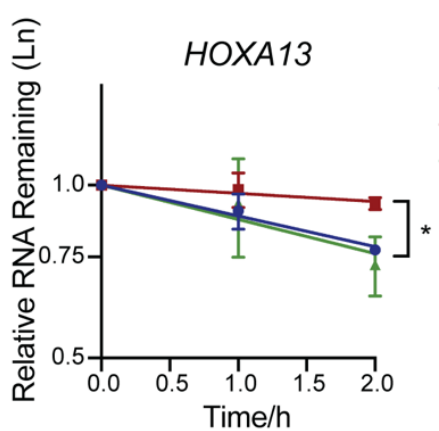

d b

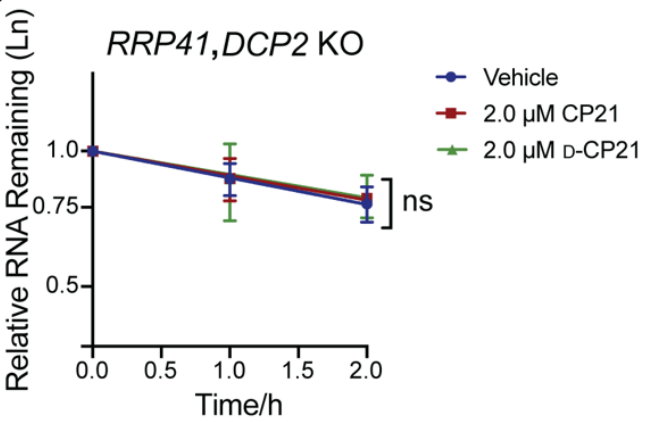

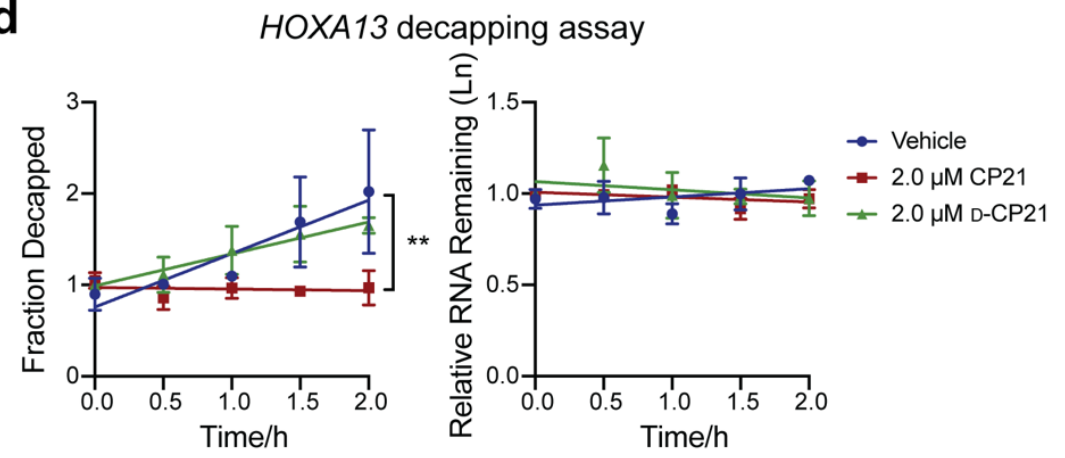
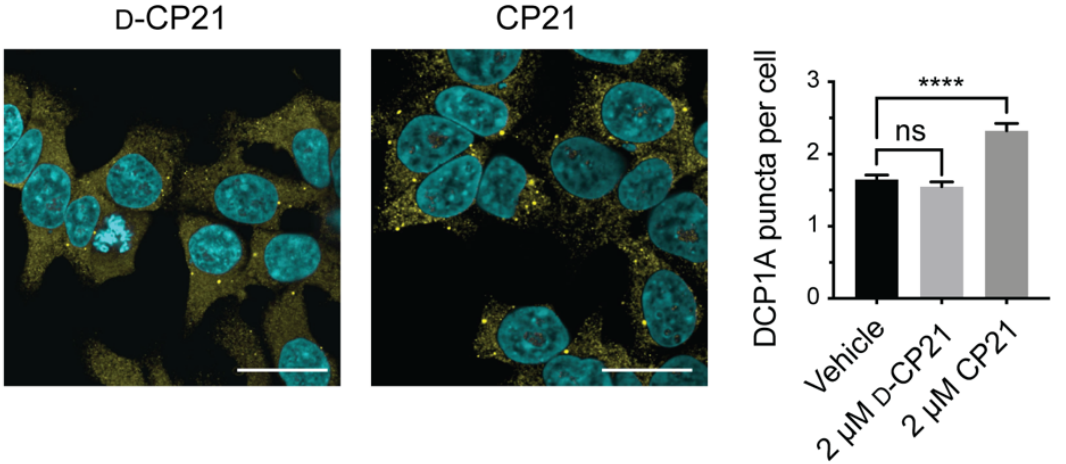

Figure 4. CP21 stabilizes specific cellular Dep2 targets at the decapping step. (a) RNA decay curves of selected Dcp2 targets from HEK293T cells treated with $2 \mu \mathrm{M}$ of CP21 (red), D-CP21 (green), or vehicle (equal volume of peptide storage buffer, blue). Relative levels of each RNA remaining at indicated time points following actinomycin D treatment were determined by quantitative reverse transcription PCR (qRT-PCR, see methods). Error bars represent mean \pm s.d. Number of biological repeats: $n=3$. Significance was derived from one-way ANOVA of the slope of regression lines; $\left(^{*}\right) \mathrm{p}<0.05$. (b) Decay of RRP41 RNA in DCP2 KO HEK293T cells treated with CP21, D-CP21, or vehicle. Number of biological repeats: $n=3$. Error bars represent \pm s.d. P-values (ANOVA linear regression with Dunnett's test) are denoted by asterisks; (ns) non-significant. (c) PLBD2, the stability of which is not affected by Dcp2, was not stabilized by CP2 1 according to qRT-PCR analysis. Number of biological repeats: $n=3$. Error bars represent \pm s.d, and significance was evaluated with ANOVA linear regression; (ns) non-significant. (d) Splinted ligation RT-PCR (qSL-RT-PCR) assay was used to detect decapped RNA under CP21 treatment. The relative fraction decapped HOXA13 transcripts following transcriptional block for the indicated times in XRN1 KO cells treated with CP21, D-CP21, or vehicle was normalized to $\mathrm{t}=0$ (left). The mean of three biological replicates was plotted. Error bars represent \pm s.d. Significance at all time points was analyzed by ANOVA linear regression (with Dunnett's test); $\left({ }^{* *}\right) \mathrm{p}<0.01$. Total RNA level of HOXA13 in XRN1 KO cells from the same samples measured by qRT-PCR suggested treatment with neither compound affects total RNA level in XRN1 KO (right). (e) P-body numbers in HEK293T cells treated with $2 \mu \mathrm{M}$ CP21, D-CP21, or the same volume of vehicle for $18 \mathrm{~h}$. Fixed cells were stained with antibodies detecting DCP1A, a P-body marker. Six fields of view ( $\geq 180$ cells) were used to quantitate average P-bodies per cell in each condition. P-body numbers were counted using ImageJ as previously described (see Methods). Data represent mean values \pm s.e.m, and significance was evaluated with one-way ANOVA (with Dunnett's test); (ns) non-significant, (****) $\mathrm{p}<0.0001$. Scale bars, $25 \mu \mathrm{m}$. 


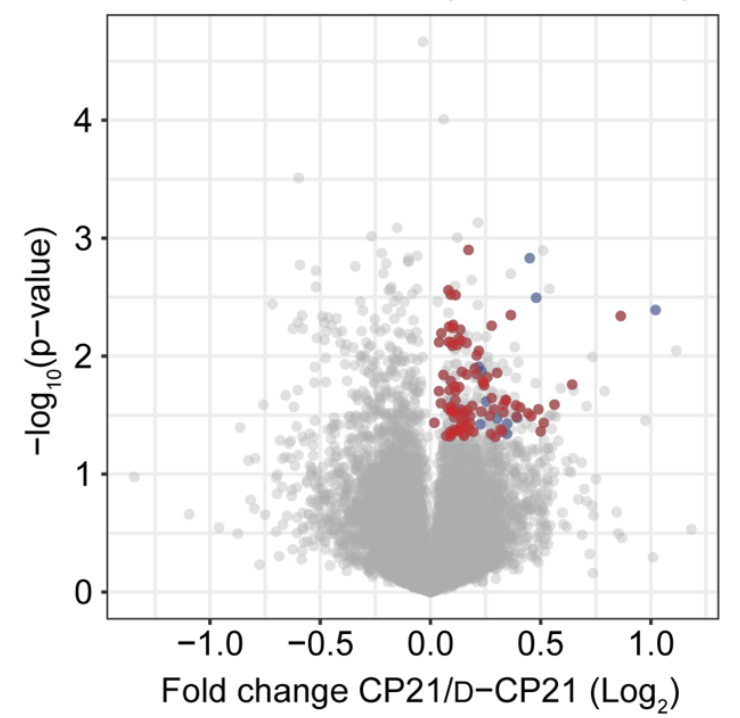

b

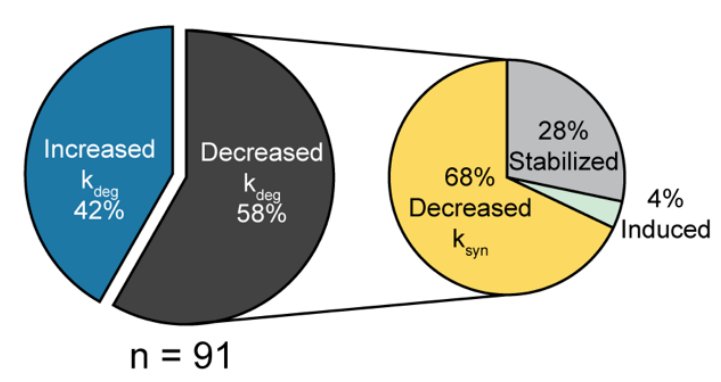

Figure 5. TimeLapse-seq profiling of CP21-stabilized RNAs enables identification of new cellular Dcp2 targets. (a) Volcano plot of TimeLapse-seq analysis ( $2 \mathrm{~h} \mathrm{~s}^{4} \mathrm{U}$ labeling period) from HEK293T cells pre-treated with $2 \mu \mathrm{M} \mathrm{CP} 21$ vs D-CP21 for 5 h. Log 2 (Fold Change) in total RNA is calculated from CP21/D-CP21, and Dcp2-dependent changes were isolated by comparing to results from $D C P 2$ KO cells under the same CP21 vs. D-CP21 treatment scheme (see Methods). Data represent two biological replicates. (b) Analysis of RNA decay rate $\left(\mathrm{k}_{\mathrm{deg}}\right)$ or synthesis rate $\left(\mathrm{k}_{\text {syn }}\right)$ changes in DCP2 KO vs WT HEK293T cells of Dcp2-dependent, CP21-stabilized RNAs. 
a
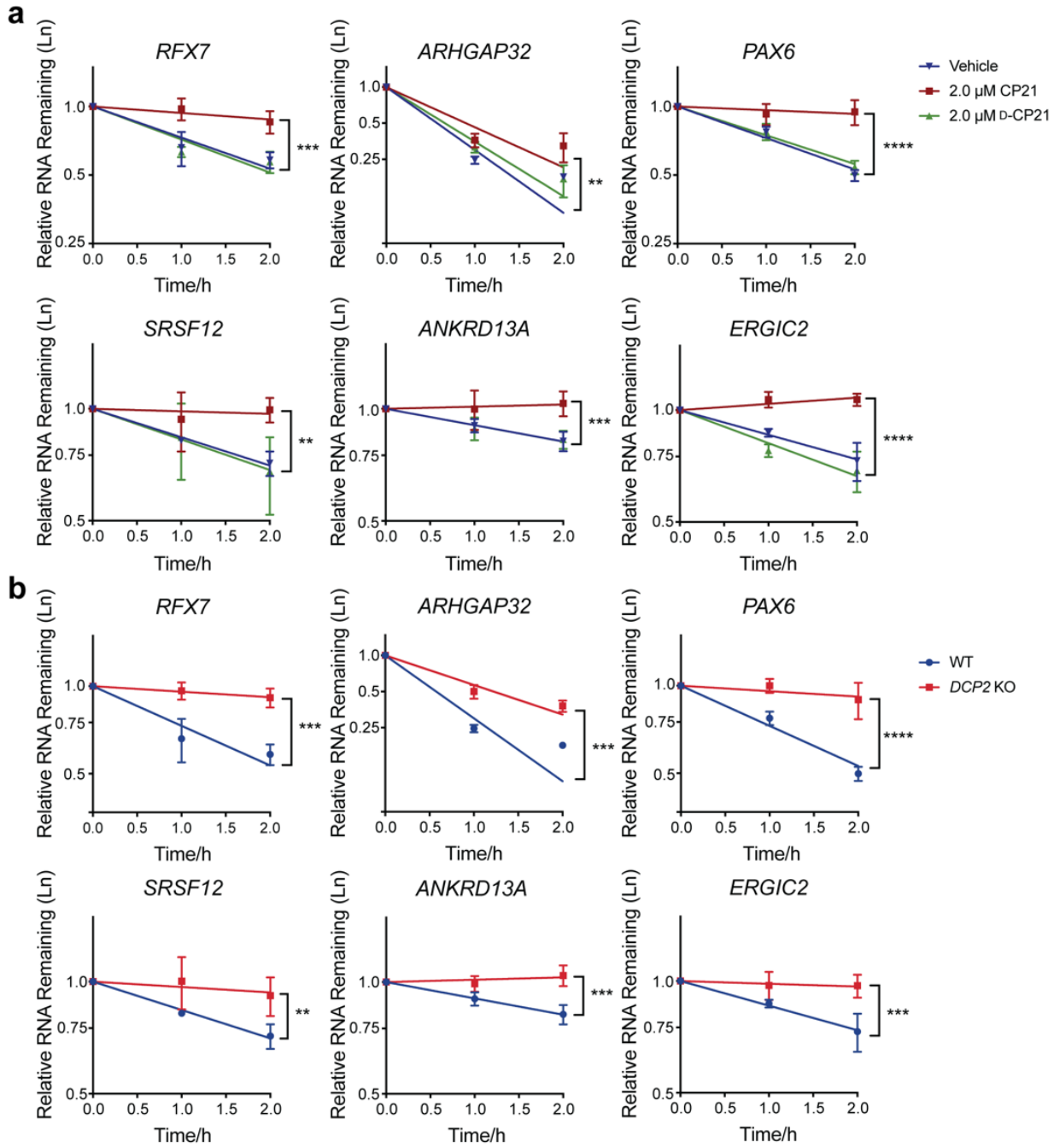

Figure 6. CP21-stabilized transcripts are cellular Dcp2 substrates. (a) The RNA lifetime of selected transcripts stabilized by CP21 were measured using transcriptional arrest and qRT-PCR. HEK293T cells were treated with $2 \mu \mathrm{M}$ of CP21 (red), D-CP21 (green), or vehicle (blue), then subjected to actinomycin D-mediated transcriptional arrest for the indicated times. Relative RNA levels were normalized to $\beta$-actin. Number of biological replicates: $n=4$. Error bars represent \pm s.d. Significance was derived from one-way ANOVA of the slope of regression lines; $(* *) \mathrm{p}<0.01 ;(* * *) \mathrm{p}<0.001 ;(* * * *) \mathrm{p}<0.0001$. (b) The stabilities of the same set of CP21 RNA targets were measured in DCP2 KO (red) vs WT (blue) HEK293T cells treated with actinomycin D for the indicated times. Number of biological replicates: $n=4$. Significance was calculated from linear regression $t$-test. 


\section{REFERENCES}

1. Y. Li, M. Kiledjian, Regulation of mRNA decapping. Wiley Interdisciplinary Rev RNA 1, 253-265 (2010).

2. T. Dunckley, R. Parker, The DCP2 protein is required for mRNA decapping in Saccharomyces cerevisiae and contains a functional MutT motif. EMBO J 18, 5411-5422 (1999).

3. Z. Wang, X. Jiao, A. Carr-Schmid, M. Kiledjian, The hDcp2 protein is a mammalian mRNA decapping enzyme. Proc Natl Acad Sci U S A 99, $12663-12668(2002)$.

4. J. Xu, J. Y. Yang, Q. W. Niu, N. H. Chua, Arabidopsis DCP2, DCP1, and VARICOSE form a decapping complex required for postembryonic development. Plant Cell 18, 3386-3398 (2006).

5. I. Behm-Ansmant et al., mRNA degradation by miRNAs and GW182 requires both CCR4:NOT deadenylase and DCP1:DCP2 decapping complexes. Genes Dev 20, 1885-1898 (2006).

6. J. Lykke-Andersen, Identification of a human decapping complex associated with hUpf proteins in nonsense-mediated decay. Mol Cell Biol $\mathbf{2 2}$, 8114-8121 (2002).

7. J. H. Yoon, E. J. Choi, R. Parker, Dcp2 phosphorylation by Ste20 modulates stress granule assembly and mRNA decay in Saccharomyces cerevisiae. J Cell Biol 189, 813-827 (2010).

8. Y. Luo, Z. Na, S. Slavoff, P-Bodies: Composition, Properties, and Functions. Biochemistry 57, 2424-2431 (2018).

9. J. S. Mugridge, J. Coller, J. D. Gross, Structural and molecular mechanisms for the control of eukaryotic 5'-3' mRNA decay. Nat Struct Mol Biol 25, 1077-1085 (2018).

10. E. Grudzien-Nogalska, M. Kiledjian, New insights into decapping enzymes and selective mRNA decay. Wiley Interdiscip Rev RNA 8, e1379 (2017).

11. M. Kiledjian, Eukaryotic RNA 5'-End NAD. Trends Cell Biol 28, 454-464 (2018).

12. Y. Li, M. Song, M. Kiledjian, Differential utilization of decapping enzymes in mammalian mRNA decay pathways. $R N A$ 17, 419-428 (2011).

13. M. G. Song, Y. Li, M. Kiledjian, Multiple mRNA decapping enzymes in mammalian cells. Mol Cell 40, 423-432 (2010).

14. E. Grudzien-Nogalska, X. Jiao, M. G. Song, R. P. Hart, M. Kiledjian, Nudt3 is an mRNA decapping enzyme that modulates cell migration. RNA 22, 773-781 (2016).

15.

X. Jiao, J. H. Chang, T. Kilic, L. Tong, M. Kiledjian, A mammalian pre-mRNA 5' end capping quality control mechanism and an unexpected link of capping to pre-mRNA processing. Mol Cell 50, 104-115 (2013).

16. J. H. Chang et al., Dxo1 is a new type of eukaryotic enzyme with both decapping and 5'-3' exoribonuclease activity. Nat Struct Mol Biol 19, 10111017 (2012).

17.

X. Jiao et al., 5' End Nicotinamide Adenine Dinucleotide Cap in Human Cells Promotes RNA Decay through DXO-Mediated deNADding. Cell 168, 1015-1027.e1010 (2017).

18. L. S. Cohen et al., Dcp2 Decaps m2,2,7GpppN-capped RNAs, and its activity is sequence and context dependent. Mol Cell Biol 25, 8779-8791 (2005).

19. Y. Li, M. G. Song, M. Kiledjian, Transcript-specific decapping and regulated stability by the human Dep2 decapping protein. Mol Cell Biol 28, 939-948 (2008).

20. Y. Luo, J. Schofield, M. D. Simon, S. A. Slavoff, Global profiling of cellular substrates of human Dcp2. Biochemistry 10.1021/acs.biochem.0c00069 (2020).

21. Y. Li, J. Dai, M. Song, P. Fitzgerald-Bocarsly, M. Kiledjian, Dcp2 decapping protein modulates mRNA stability of the critical interferon regulatory factor (IRF) IRF-7. Mol Cell Biol 32, 1164-1172 (2012).

22. X. Wang et al., N6-methyladenosine-dependent regulation of messenger RNA stability. Nature 505, 117-120 (2014).

23. E. Valkov et al., Structure of the Dcp2-Dcp1 mRNA-decapping complex in the activated conformation. Nat Struct Mol Biol 23, 574-579 (2016).

24. C. Charenton et al., Structure of the active form of Dcp1-Dcp2 decapping enzyme bound to m7GDP and its Edc3 activator. Nat Struct Mol Biol 23, 982-986 (2016).

25. J. S. Mugridge, M. Ziemniak, J. Jemielity, J. D. Gross, Structural basis of mRNA-cap recognition by Dep1-Dcp2. Nat Struct Mol Biol 23, 987-994 (2016).

26. J. S. Mugridge, R. W. Tibble, M. Ziemniak, J. Jemielity, J. D. Gross, Structure of the activated Edc1-Dcp1-Dcp2-Edc3 mRNA decapping complex with substrate analog poised for catalysis. Nat Commun 9, 1152 (2018).

27. J. P. Wurm, I. Holdermann, J. H. Overbeck, P. H. O. Mayer, R. Sprangers, Changes in conformational equilibria regulate the activity of the Dep2 decapping enzyme. Proc Natl Acad Sci U S A 114, 6034-6039 (2017).

28. D. R. Paquette, R. W. Tibble, T. S. Daifuku, J. D. Gross, Control of mRNA decapping by autoinhibition. Nucleic Acids Res $10.1093 /$ nar/gky233 (2018).

29. M. She et al., Structural basis of dep2 recognition and activation by dcp1. Mol Cell 29, 337-349 (2008).

30. C. T. Chang, N. Bercovich, B. Loh, S. Jonas, E. Izaurralde, The activation of the decapping enzyme DCP2 by DCP1 occurs on the EDC4 scaffold and involves a conserved loop in DCP1. Nucleic Acids Res 42, 5217-5233 (2014).

31. F. Tritschler et al., DCP1 forms asymmetric trimers to assemble into active mRNA decapping complexes in metazoa. Proc Natl Acad Sci U S A 106, 21591-21596 (2009).

32. C. T. Chang et al., A low-complexity region in human XRN1 directly recruits deadenylation and decapping factors in 5'-3' messenger RNA decay. Nucleic Acids Res 10.1093/nar/gkz633 (2019).

33. M. Ziemniak et al., Two-headed tetraphosphate cap analogs are inhibitors of the Dcp1/2 RNA decapping complex. RNA 22, 518-529 (2016).

34. M. Arribas-Layton, D. Wu, J. Lykke-Andersen, H. Song, Structural and functional control of the eukaryotic mRNA decapping machinery. Biochim Biophys Acta 1829, 580-589 (2013).

35.

36. W. S. Horne, T. N. Grossmann, Proteomimetics as protein-inspired scaffolds with defined tertiary folding patterns. Nat Chem 12, 331-337 (2020). L. D. Walensky, G. H. Bird, Hydrocarbon-stapled peptides: principles, practice, and progress. J Med Chem 57, 6275-6288 (2014).

K. Deyle, X. D. Kong, C. Heinis, Phage Selection of Cyclic Peptides for Application in Research and Drug Development. Acc Chem Res 50, 18661874 (2017).

38. A. A. Vinogradov, Y. Yin, H. Suga, Macrocyclic Peptides as Drug Candidates: Recent Progress and Remaining Challenges. J Am Chem Soc 141, 4167-4181 (2019).

39. L. Dietrich et al., Cell Permeable Stapled Peptide Inhibitor of Wnt Signaling that Targets $\beta$-Catenin Protein-Protein Interactions. Cell Chem Biol 24, 958-968.e955 (2017).

40. J. R. LaRochelle, G. B. Cobb, A. Steinauer, E. Rhoades, A. Schepartz, Fluorescence correlation spectroscopy reveals highly efficient cytosolic delivery of certain penta-arg proteins and stapled peptides. J Am Chem Soc 137, 2536-2541 (2015).

41. C. Heinis, T. Rutherford, S. Freund, G. Winter, Phage-encoded combinatorial chemical libraries based on bicyclic peptides. Nat Chem Biol 5, 502507 (2009).

42. A. Angelini et al., Bicyclic peptide inhibitor reveals large contact interface with a protease target. ACS Chem Biol 7, 817-821 (2012).

43. I. Rentero Rebollo, C. Heinis, Phage selection of bicyclic peptides. Methods 60, 46-54 (2013).

44. M. Fairhead, M. Howarth, Site-specific biotinylation of purified proteins using BirA. Methods Mol Biol 1266, 171-184 (2015). 
P. J. Schatz, Use of peptide libraries to map the substrate specificity of a peptide-modifying enzyme: a 13 residue consensus peptide specifies biotinylation in Escherichia coli. Biotechnology (N Y) 11, 1138-1143 (1993).

46. C. Piccirillo, R. Khanna, M. Kiledjian, Functional characterization of the mammalian mRNA decapping enzyme hDcp2. RNA 9, $1138-1147$ (2003).

47. S. Jonas, E. Izaurralde, The role of disordered protein regions in the assembly of decapping complexes and RNP granules. Genes Dev 27, 2628$2641(2013)$.

48. Q. Chu et al., Towards understanding cell penetration by stapled peptides. MedChemComm 6, 111-119 (2015).

49. R. Jafari et al., The cellular thermal shift assay for evaluating drug target interactions in cells. Nat Protoc 9, 2100-2122 (2014).

50. N. Blewett, J. Coller, A. Goldstrohm, A quantitative assay for measuring mRNA decapping by splinted ligation reverse transcription polymerase chain reaction: qSL-RT-PCR. RNA 17, 535-543 (2011).

51. A. Eulalio, I. Behm-Ansmant, E. Izaurralde, P bodies: at the crossroads of post-transcriptional pathways. Nat Rev Mol Cell Biol 8, 9-22 (2007).

52. U, Sheth and R. Parker, Decapping and Decay of messenger rna occur in cytoplasmic processing bodies. Science 300, 805-8 (2003).

53. A. Aizer et al., Quantifying mRNA targeting to P-bodies in living human cells reveals their dual role in mRNA decay and storage. $J$ Cell Sci 127, 4443-4456 (2014).

54. M. Courel et al., GC content shapes mRNA storage and decay in human cells. Elife 8, e49708 (2019).

55. A. Hubstenberger et al., P-Body Purification Reveals the Condensation of Repressed mRNA Regulons. Mol Cell 68, 144-157.e145 (2017).

56. F. He, A. Celik, C. Wu, A. Jacobson, General decapping activators target different subsets of inefficiently translated mRNAs. Elife 7, e34409 (2018).

57. M. Howarth, K. Takao, Y. Hayashi, A. Y. Ting, Targeting quantum dots to surface proteins in living cells with biotin ligase. Proc Natl Acad Sci US A 102, 7583-7588 (2005).

58. S. W. Liu, X. Jiao, S. Welch, M. Kiledjian, Analysis of mRNA decapping. Methods Enzymol 448, 3-21 (2008).

59. G. H. Bird, F. Bernal, K. Pitter, L. D. Walensky, Synthesis and biophysical characterization of stabilized alpha-helices of BCL-2 domains. Methods Enzymol 446, 369-386 (2008)

60. J. Cox, M. Mann, MaxQuant enables high peptide identification rates, individualized p.p.b.-range mass accuracies and proteome-wide protein quantification. Nat Biotechnol 26, 1367-1372 (2008).

61. S. Tyanova et al., The Perseus computational platform for comprehensive analysis of (prote)omics data. Nat Methods 13, 731-740 (2016).

62. T. D. Schmittgen, K. J. Livak, Analyzing real-time PCR data by the comparative C(T) method. Nat Protoc 3, 1101-1108 (2008).

63. M. Fenger-Gron, C. Fillman, B. Norrild, J. Lykke-Andersen, Multiple processing body factors and the ARE binding protein TTP activate mRNA decapping. Mol Cell 20, 905-915 (2005).

64. T. Nissan, R. Parker, Analyzing P-bodies in Saccharomyces cerevisiae. Methods Enzymol 448, 507-520 (2008).

65. J. A. Schofield, E. E. Duffy, L. Kiefer, M. C. Sullivan, M. D. Simon, TimeLapse-seq: adding a temporal dimension to RNA sequencing through nucleoside recoding. Nat Methods 15, 221-225 (2018). 


\section{Supporting Figures}

a

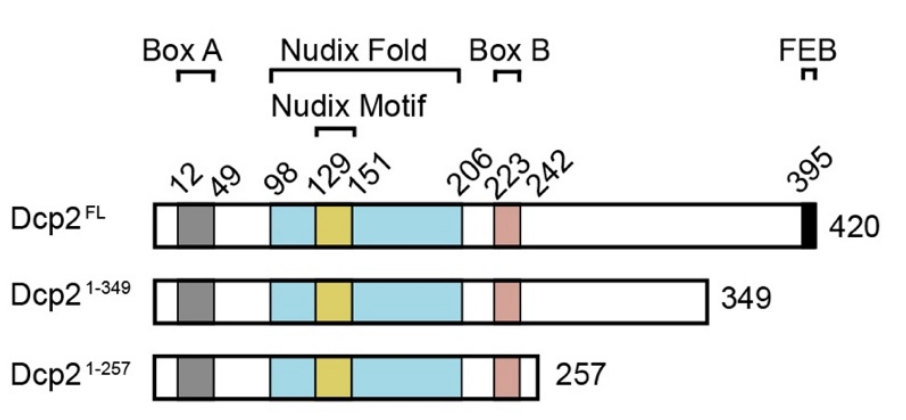

C

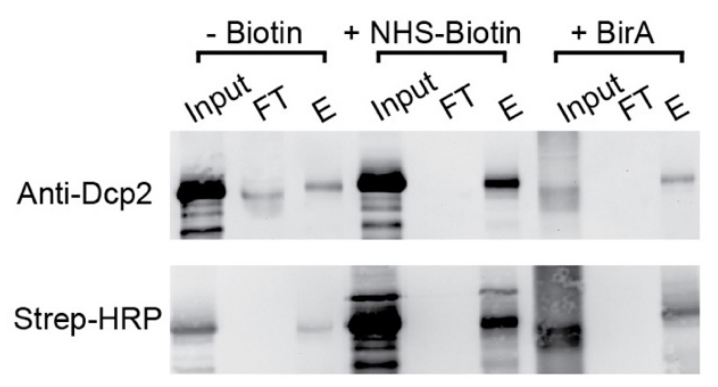

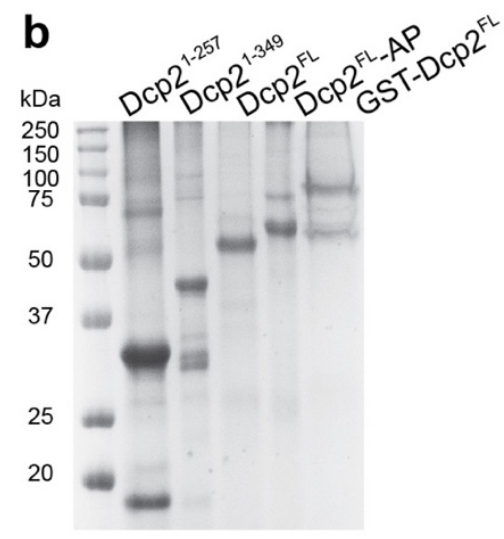

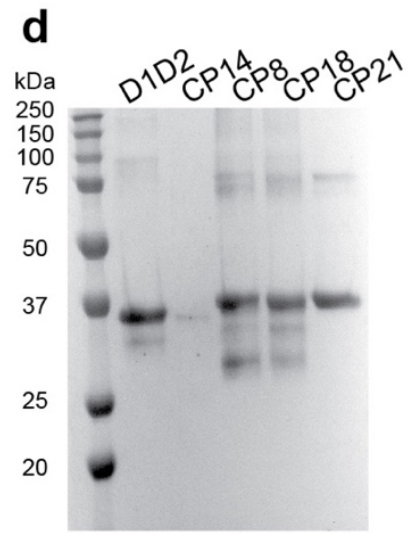

Figure S1. Purification and biotinylation of fusion proteins for affinity selection and pull-downs. (a) Schematic representation of fulllength human Dcp2 (Dcp2 ${ }^{\mathrm{FL}}$ ) and two previously reported catalytically active Dcp2 truncations (Piccirillo et al., 2003). The three conserved functional motifs, Box A (gray), catalytic Nudix motif (yellow) within the Nudix fold (cyan), and Box B (pink) are highlighted. The short C-terminal disordered phenylalanine-rich EDC4 binding (FEB) motif responsible for EDC4 binding (Chang et al., 2014) is shown in black. All proteins contain a C-terminal His 6 tag for purification. For some experiments, AviTag peptide (AP) was added by inverse PCR to the Cterminus, and a GST fusion construct was appended to the N-terminus (see Methods). Numbers represent amino acid positions. (b) SDSPAGE analysis of all Dcp2 truncations and tagged full-length proteins used in this study (12\% polyacrylamide gel stained with Coomassie blue). (c) Confirmation of in vitro BirA biotinylation of Dcp2 $2^{\mathrm{FL}}$-AP by streptavidin pull-down assay. Aliquots of 1 bead volume of each biotinylation reaction were mixed with $20 \mu \mathrm{L}$ of streptavidin magnetic beads and incubated at room temperature for 30 min. The bound fractions were eluted by boiling in SDS sample buffer for $20 \mathrm{~min}$ and loaded on $10 \%$ acrylamide gel together with the supernatant (FT) and input for analysis by western blot. (d) SDS-PAGE analysis of FLAG-tagged D1D2 domain of phage protein pIII and the four TBMB-cyclized peptide (CP)-D1D2 fusions used for the pull-down experiment in Figure 2b. The proteins were stained with Coomassie blue. 


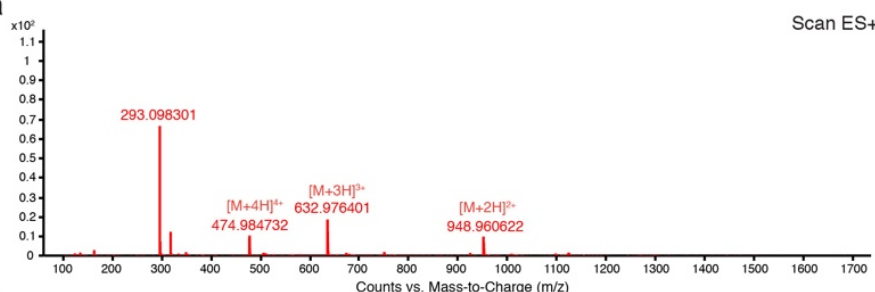

b
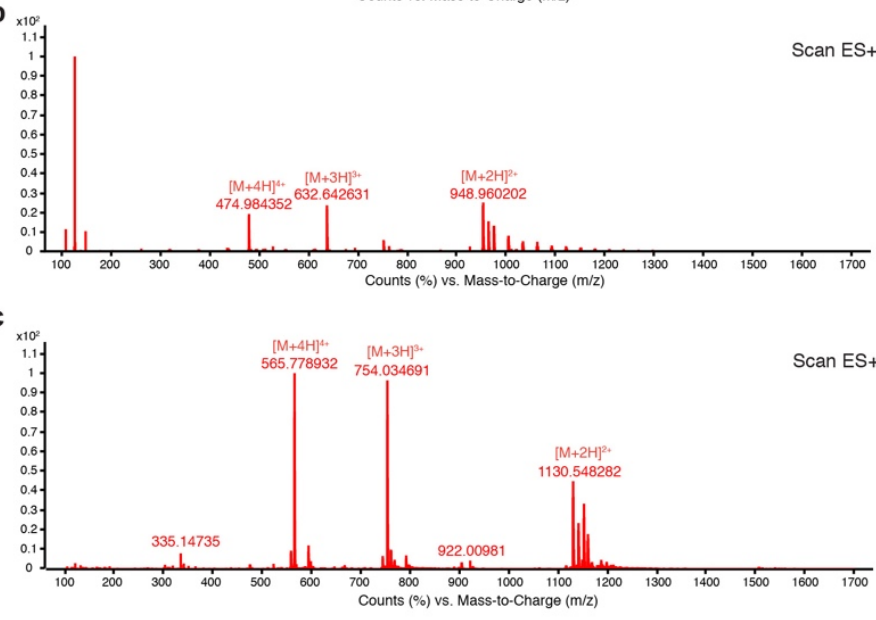

d
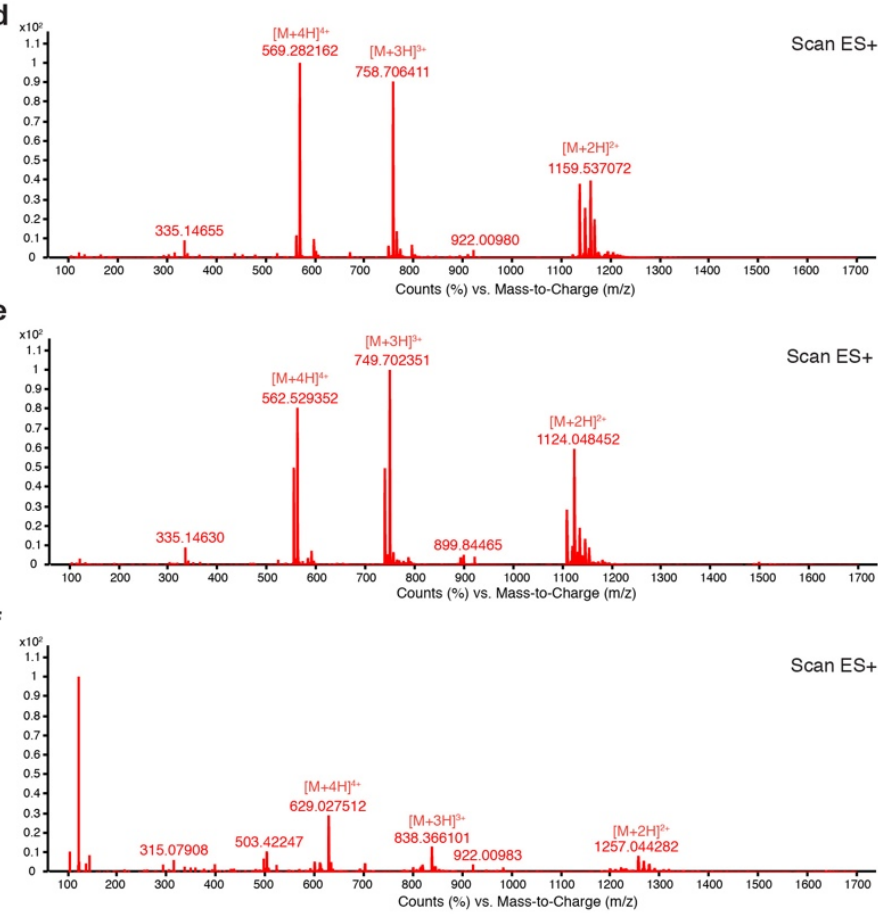

g

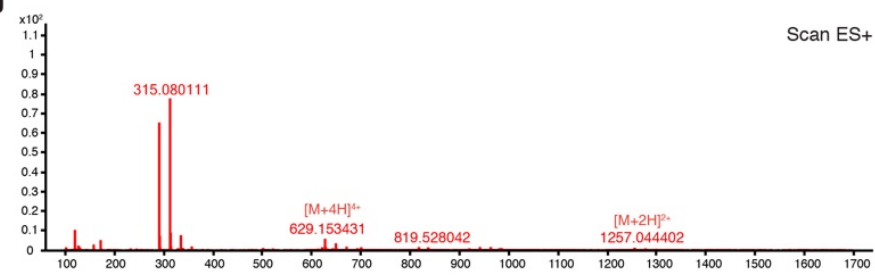

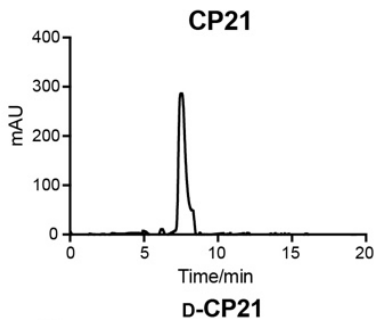

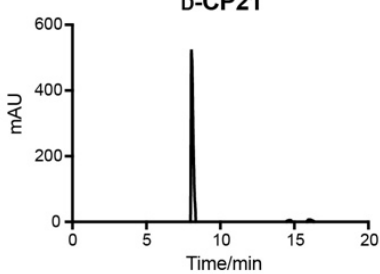

Photo-CP-1

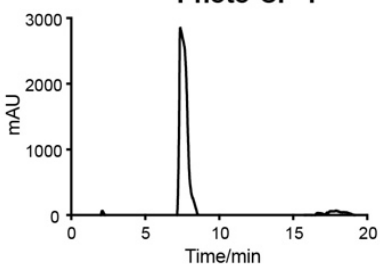

Photo-CP-2
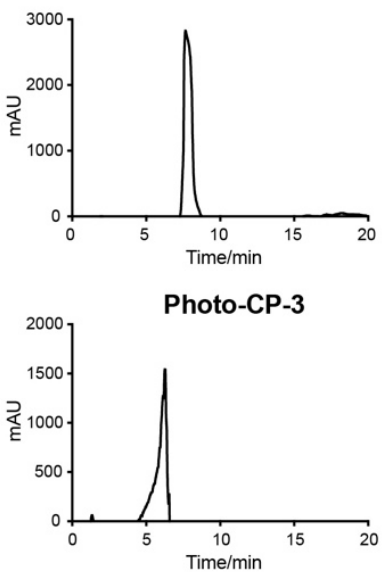

FITC-Ahx-CP21

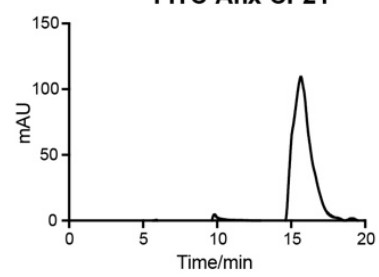

FITC-Ahx-D-CP21

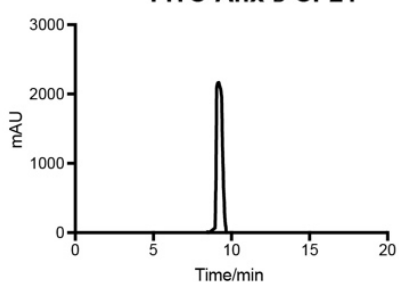

Figure S2. LC/MS and HPLC traces for all chemically synthesized peptides used in this study. (a) CP21, (b) all-D CP21 (D-CP21), (ce) Photo-cross-linkable CP21 analogue (Photo-CP-1 to -3), (f) FITC-Ahx-CP21 and (g) all-D FITC-Ahx-CP21 (FITC-Ahx-D-CP21) in storage buffer were diluted $1 / 50$ in $\mathrm{ddH}_{2} \mathrm{O}$ for liquid chromatography-mass spectrometry (LC/MS) analysis (left panels) to confirm purified product masses. HPLC traces (right panels) show purity. 
a

Photo-CP-1 ACPK(Photo-L)HPPCSKPKTQCG

Photo-CP-2 ACPKTHPPC(Photo-L)KPKTQCG

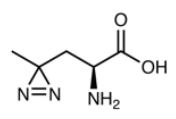

Photo-L

Photo-CP-3 ACPKTHPPCSKPKT(Photo-M)CG

b
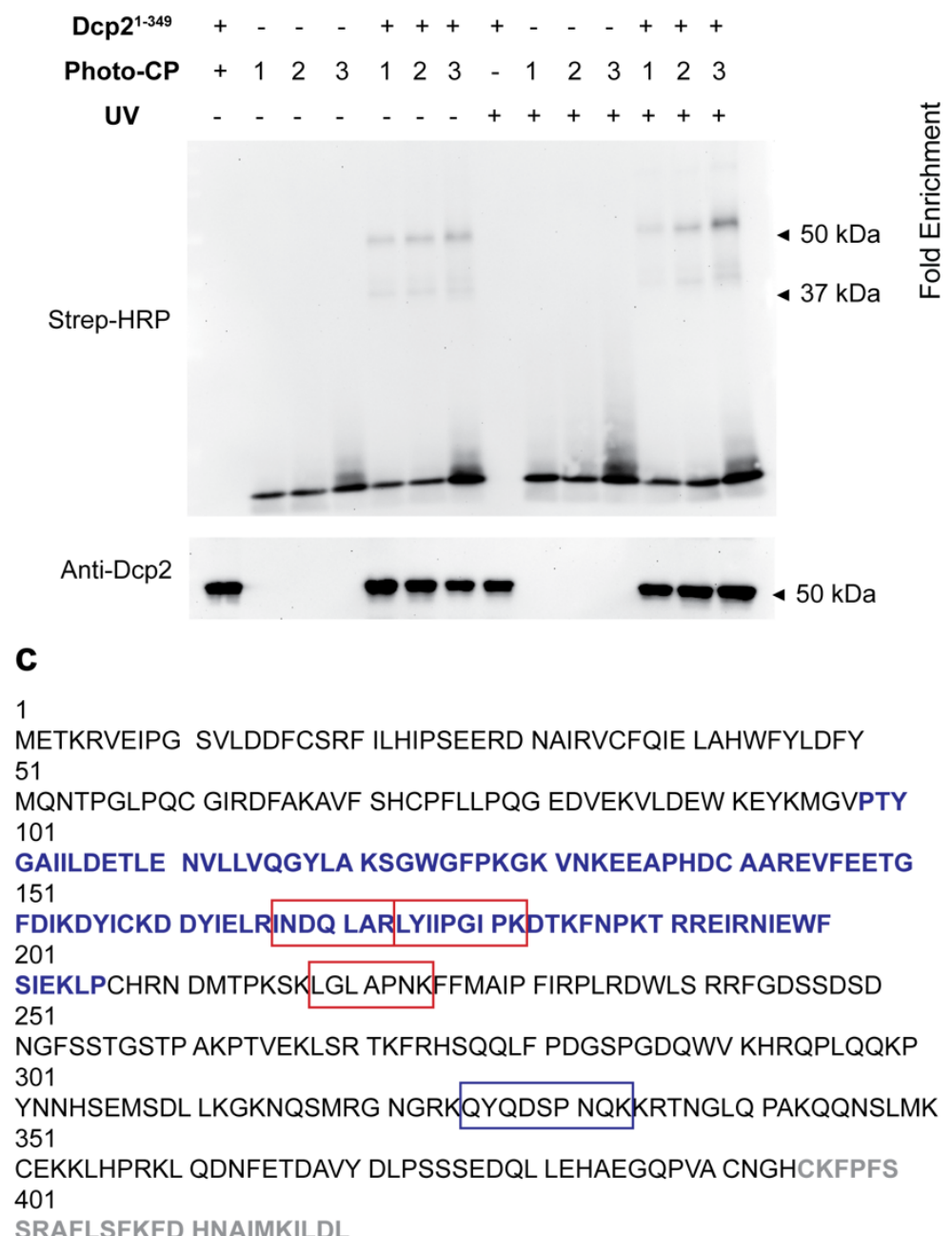

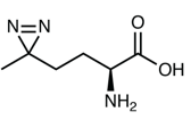

Photo-M

d

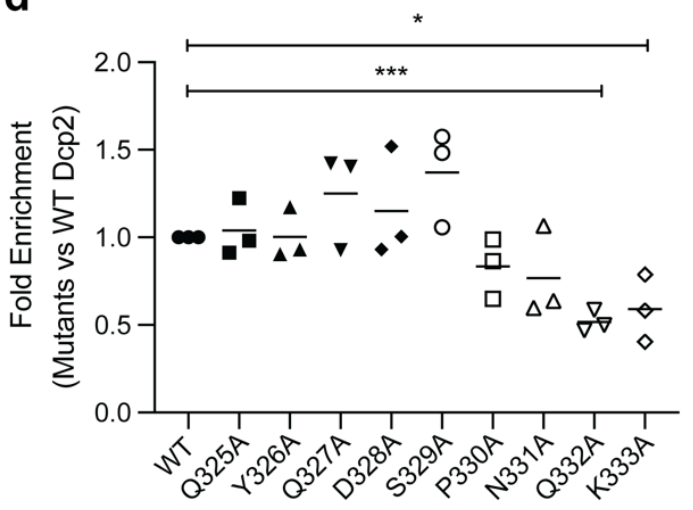

Figure S3. A segment of the C-terminus disordered region of Dcp2 is involved in CP21 binding. (a) Design of photo-cross-linkable CP21 probes. L-Photo-Methionine (Photo-M) or L-Photo-Leucine (Photo-L) were incorporated into CP21 during synthesis at three residues not conserved from prior selection result. (b) Photo-cross-linking of each CP21 probe with purified Dcp2 $2^{1-349}$ at $365 \mathrm{~nm}$. A higher molecular weight cross-linked product was observed near the molecular weight of Dcp2. (c) Respective regions of Dcp2 photo-cross-linked to PhotoCP-3. Boxes indicated the four fragments consistently missing from UV irradiated samples vs unirradiated control are emphasized in boxes (red, observed in at least two out of three of the unirradiated control replicates; blue, additionally validated by in vitro pull-down). Experiments were performed three times independently. The Nudix domain are highlighted in blue and the FEB motif in grey. (d) Alanine scanning mutagenesis of Dcp2 between amino acid residues 325 and 333 was performed to identify residues directly interacting with CP21. GST-tagged wild-type full length Dep2 or the indicated mutants were separately co-incubated with 10-fold molar excess of the FLAG epitope-tagged CP21-D1D2 fusion proteins, with CP21-D1D2 alone as a negative control, and GST pull-down was performed prior to western blot detection. Lines from the scatter plot indicate the mean value of CP21-D1D2 enrichment using each Dcp2 mutant comparing to the wild-type protein. 


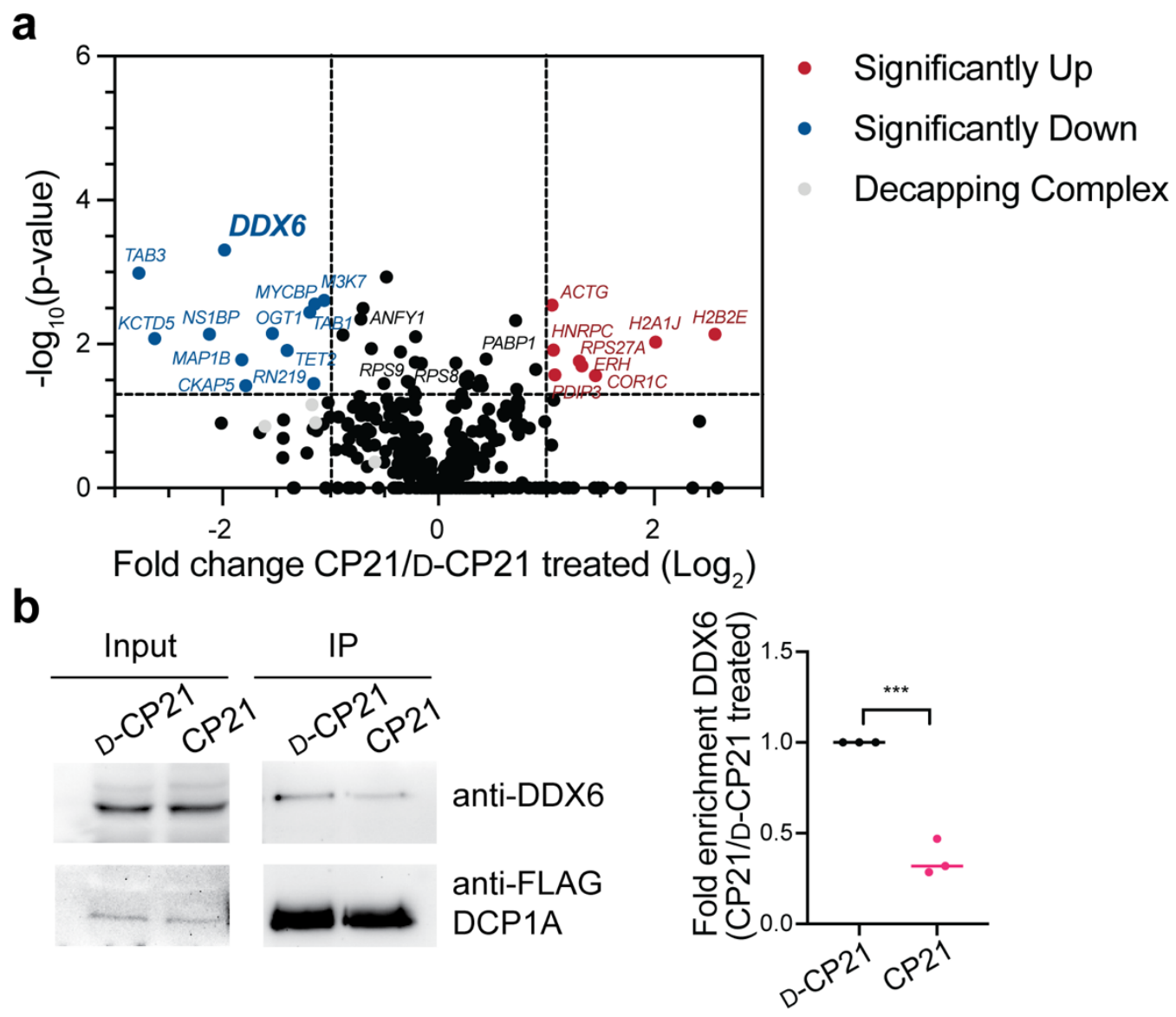

Figure S4. CP21 regulates the interaction between RNA decapping factors. RNA decapping complexes were purified from HEK293T cells stably expressing FLAG-DCP1A after pre-treatment with CP21 vs D-CP21 (2 $\mu \mathrm{M}, 18 \mathrm{~h})$. Mass spectrometry analysis was performed to identify difference in the composition of immunopurified decapping complex (a). The most depleted protein, DDX6, in CP21 vs D-CP21treated groups was further validated by western blotting (b). Data represent three biological replicates. Lines from the scatter plot (b, right) indicate the mean value of DDX6 band intensity normalized to that of DCP1A probed using anti-FLAG. 
a

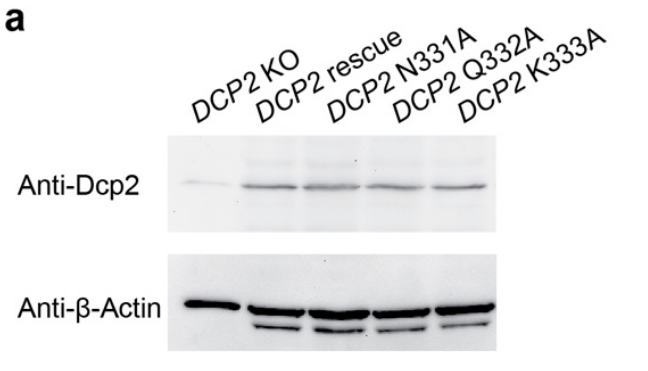

C

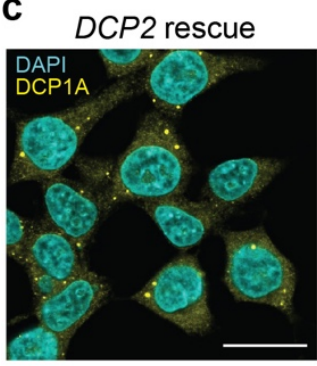

$D C P 2 \mathrm{~K} 333 \mathrm{~A}$
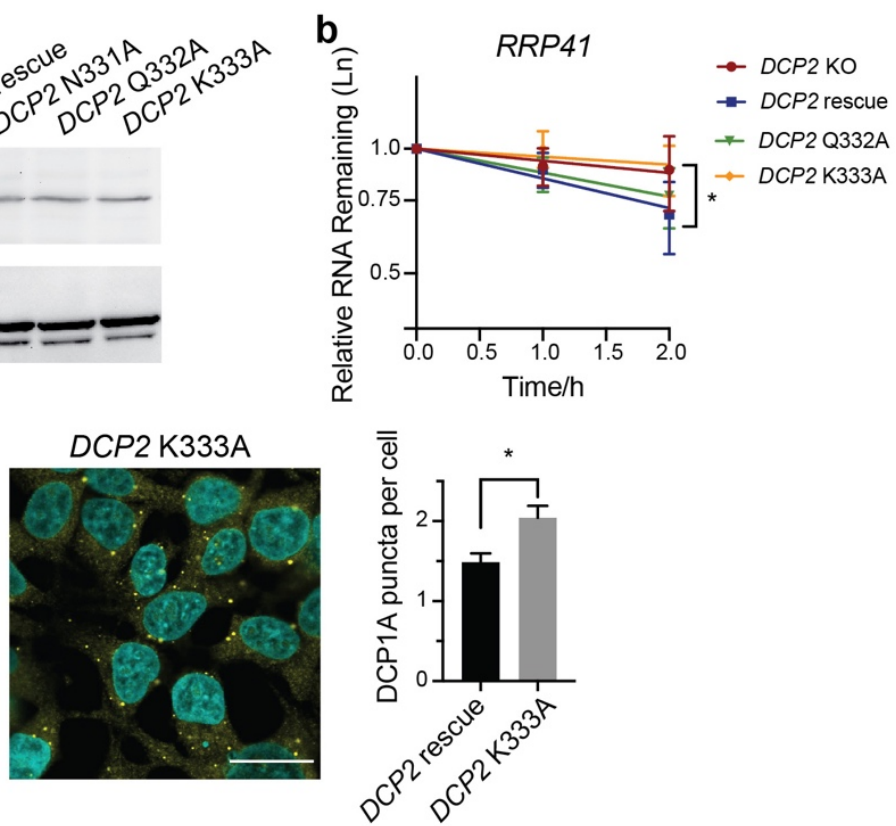

Figure S5. K333 is important for CP21 binding and human Dep2 function. (a) Validation of DCP2 rescue HEK293T and mutant complementation cell lines by western blot. (b) RRP41 RNA decay curves from $D C P 2$ knockout (KO) HEK293T cells versus $D C P 2$ rescue or two non-CP21-interacting mutant Dcp2 complementation cell lines. The levels of RRP41 RNA remaining following actinomycin D treatment at indicated time points relative to $\mathrm{t}=0 \mathrm{~h}$ were determined by qRT-PCR. Significance was analyzed by ANOVA linear regression. Number of biological repeats: $n=4$. P-values with Dunnett's test are denoted by asterisks; $(*) \mathrm{p}<0.05$. (c) P-body numbers in $D C P 2$ rescue versus $D C P 2 \mathrm{~K} 333 \mathrm{~A}$ complementation cell line. Fixed cells were stained with antibodies detecting DCP1A, a P-body marker. At least 300 cells from six fields of view were used to quantitate average P-bodies per cell in each condition. P-body numbers were counted using ImageJ (see Methods). Data represent mean values \pm s.e.m. Significance was evaluated with two-tailed $t$-test; $(*) \mathrm{p}<0.05$. Scale bars, $25 \mu \mathrm{m}$. 


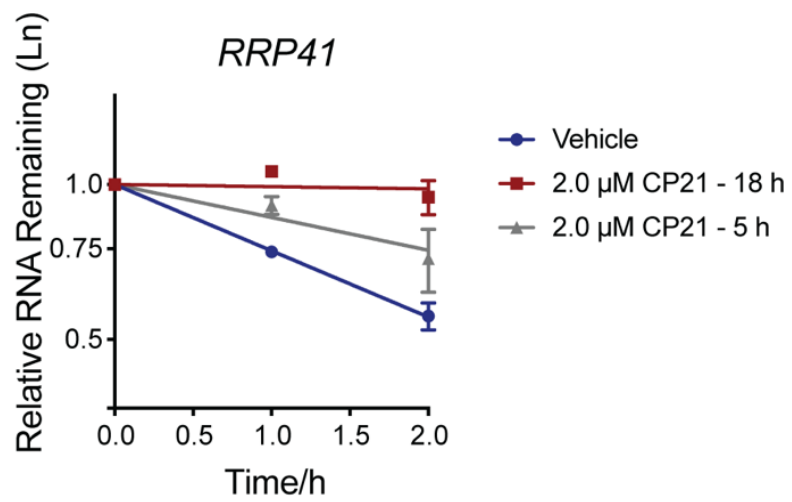

Figure S6. Validation of CP21 treatment scheme for TimeLapse-seq experiments. RRP41 RNA decay curves of HEK293T cells treated with $2 \mu \mathrm{M} \mathrm{CP} 21$ for $5 \mathrm{~h}$ vs $18 \mathrm{~h}$. The levels of RRP41 RNA remaining following actinomycin D treatment at indicated time points relative to $\mathrm{t}=0 \mathrm{~h}$ were determined by qRT-PCR, with $\mathrm{Ct}$ value normalized to U6 based on previous reports(19). Number of biological repeats: $n=2$. 


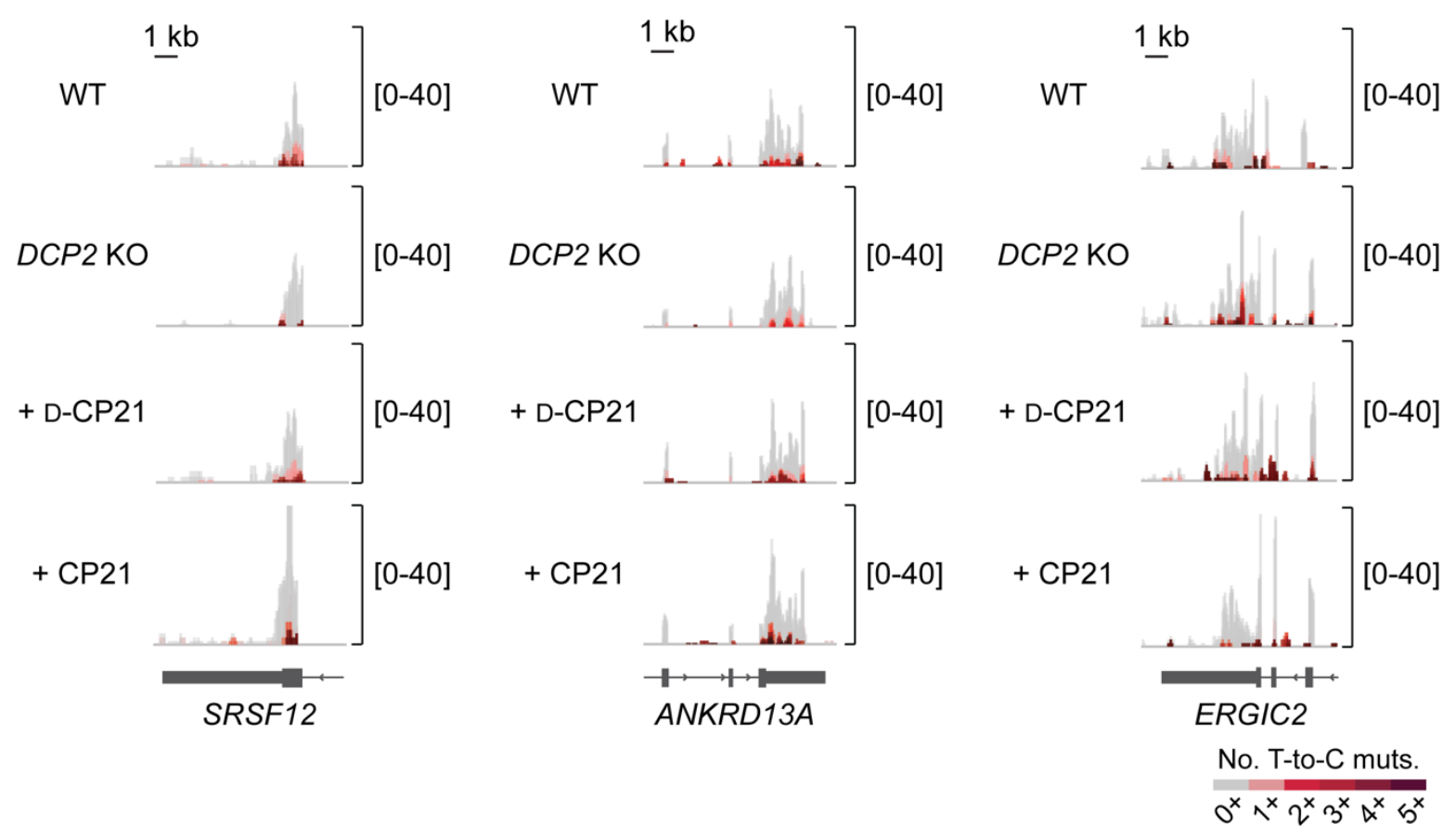

Figure S7. TimeLapse-seq tracks depicting examples of authentic Dcp2 targets missing from a prior study based on $D C P 2$ genetic knock out due to secondary changes in RNA synthesis. TimeLapse-seq tracks of three newly identified Dcp2 targets in HEK293T cells pretreated with CP21 vs D-CP21 $(2 \mu \mathrm{M}, 5 \mathrm{~h})$ were compared to that in untreated WT vs DCP2 KO HEK293T cells. Increasing T-to-C mutational content indicates higher recoded $\mathrm{s} U$ incorporation over the $2 \mathrm{~h}$ metabolic labeling period for newly synthesized mature RNA. 
Table S1. Genes specifically stabilized by CP21 vs D-CP21 via Dcp2

\begin{tabular}{|c|c|}
\hline Genes & Gene Descriptions \\
\hline ABR & Homo sapiens active BCR-related \\
\hline AES & Homo sapiens amino-terminal enhancer of split \\
\hline AGBL5 & Homo sapiens ATP/GTP binding protein like 5 \\
\hline ALG10 & Homo sapiens alpha-1,2-glucosyltransferase \\
\hline ANKRD13A & Homo sapiens ankyrin repeat domain $13 \mathrm{~A}$ \\
\hline ARFRP1 & Homo sapiens ADP ribosylation factor related protein 1 \\
\hline ARHGAP32 & Homo sapiens Rho GTPase activating protein 32 \\
\hline ARMCX3 & Homo sapiens armadiollo repeat containing, $\mathrm{X}$-linked 3 \\
\hline ATP11A & Homo sapiens ATPase phospholipid transporting 11A \\
\hline BCS1L & $\begin{array}{l}\text { Homo sapiens BCS1 homolog, ubiquinol-cytochromo c reductase complex } \\
\text { chaperone }\end{array}$ \\
\hline BUB1B & Homo sapiens $\mathrm{BUB} 1$ mitotic checkpoint serine/threonine kinase B \\
\hline C18orf21 & Homo sapiens chromosome 18 open reading frame 21 \\
\hline CABYR & Homo sapiens calcium binding tyrosine phosphorylation regulated \\
\hline CARHSP1 & Homo sapiens calcium regulated heat stable protein 1 \\
\hline CCT8 & Homo sapiens chaperonin containing TCP1 subunit 8 \\
\hline CEP290 & Homo sapiens centrosomal protein 290 \\
\hline CHUK & Homo sapiens conserved helix-loop-helix ubiquitous kinase \\
\hline COPS2 & Homo sapiens COP9 signalosome subunit 2 \\
\hline DDX17 & Homo sapiens DEAD-box helicase 17 \\
\hline DDX39B & Homo sapiens DEAD-box helicase 39B \\
\hline EML4 & Homo sapiens echinoderm microtubule associated protien like 4 \\
\hline EPPK1 & Homo sapiens epiplakin 1 \\
\hline ERGIC2 & Homo sapiens ERGIC and golgi2 \\
\hline ERO1A & Homo sapiens endoplasmic reticulum oxidoreductase 1 alpha \\
\hline EXD2 & Homo sapiens exonuclease $3^{\prime}-5^{\prime}$ domain containing 2 \\
\hline FAM168B & Homo sapiens family with sequence similarity 168 member B \\
\hline FANCB & Homo sapiens Fanconi anemia complementation group B \\
\hline FNTA & Homo sapiens farnesyltransferase, CAAX box, alpha \\
\hline FUNDC2 & Homo sapiens FUN14 domain containing 2 \\
\hline GINS4 & Homo sapiens GINS complex subunit 4 \\
\hline GRHPR & Homo sapiens glyoxylate and hydroxypyruvate reductase \\
\hline HARS & Homo sapiens histidyl-tRNA synthetase \\
\hline
\end{tabular}




\begin{tabular}{|c|c|}
\hline HCCS & Homo sapiens holocytochrome c synthase \\
\hline HNRNPA0 & Homo sapiens heterogeneous nuclear ribonucleoprotein A0 \\
\hline HS6ST1P1 & Homo sapiens heparan sulfate $6-O$-sulfotransferase 1 pseudogene 1 \\
\hline HSD17B7 & Homo sapiens hydroxysteroid 17-beta dehydrogenase 7 \\
\hline ILKAP & Homo sapiens ILK associated serine/threonine phosphatase \\
\hline INPP5A & Homo sapiens inositol polyphosphate-5-phosphatase A \\
\hline JOSD1 & Homo sapiens Josephin domain containing 1 \\
\hline KHNYN & Homo sapiens $\mathrm{KH}$ and NYN domain containing \\
\hline KLHL17 & Homo sapiens kelch like family member 17 \\
\hline LMF2 & Homo sapiens lipase maturation factor 2 \\
\hline LSM6 & $\begin{array}{l}\text { Homo sapiens LSM6 homolog, U6 small nuclear RNA and mRNA degradation } \\
\text { associated }\end{array}$ \\
\hline MBD1 & Homo sapiens methyl-CpG binding domain protein 1 \\
\hline MEGF8 & Homo sapiens multiple EGF like domains 8 \\
\hline ММАСНC & $\begin{array}{l}\text { Homo sapiens methylmalonic aciduria (cobalamin deficiency) cblC type, with } \\
\text { homocystinuria }\end{array}$ \\
\hline MOCS2 & Homo sapiens molybdenum cofactor synthesis 2 \\
\hline MRPL14 & Homo sapiens mitochondrial ribosomal protein L14 \\
\hline MSH3 & Homo sapiens mutS homolog 3 \\
\hline NBPF14 & Homo sapiens neuroblastoma breakpoint family member 14 \\
\hline NOL4L & Homo sapiens nucleolar protein 4 like \\
\hline ODC1 & Homo sapiens ornithine decarboxylase 1 \\
\hline OGFR & Homo sapiens opioid growth factor receptor \\
\hline PAGR1 & Homo sapiens PAXIP1 associated glutamate rich protein 1 \\
\hline PARS2 & Homo sapiens prolyl-tRNA synthetase 2 , mitochondrial \\
\hline PAX6 & Homo sapiens paired box 6 \\
\hline PDHB & Homo sapiens pyruvate dehydrogenase (lipoamide) beta \\
\hline PEPD & Homo sapiens peptidase D \\
\hline PET100 & Homo sapiens PET100 homolog \\
\hline PIAS2 & Homo sapiens protein inhibitor of activated STAT2 \\
\hline PLEKHJ1 & Homo sapiens pleckstrin homology domain containing $\mathrm{J} 1$ \\
\hline PPP4R3A & Homo sapiens protein phosphatase 4 regulatory subunit $3 \mathrm{~A}$ \\
\hline PSMF1 & Homo sapiens proteasome inhibitor subunit 1 \\
\hline PUSL1 & Homo sapiens pseudouridylate synthase-like 1 \\
\hline RAVER1 & Homo sapiens ribonucleoprotein, PTB binding 1 \\
\hline
\end{tabular}


RFX7

RIMKLA

RP11-295P9.3

RPAP2

SAPCD2

SDHB

SIAH1

SLC35A4

SMAP1

SOCS6

SRSF12

SRSF7

STAG3L2

STK25

TES

TFDP2

TPM1

TRAF4

TRIM8

TSPAN33

UGP2

UQCR10

ZDHHC3

ZNF180

ZNF432

ZRANB3
Homo sapiens regulatory factor X7

Homo sapiens ribosomal modification protein rimK like family member A

Homo sapiens pre-mRNA processing factor 31 (PRPF31)

Homo sapiens RNA polymerase II associated protein 2

Homo sapiens suppressor APC domain containing 2

Homo sapiens succinate dehydrogenase complex iron sulfur subunit B

Homo sapiens siah E3 ubiquitin protein ligase 1

Homo sapiens solute carrier family 35 member A4

Homo sapiens small ArfGAP1

Homo sapiens suppressor of cytokine signaling 6

Homo sapiens serine and arginine rich splicing factor 12

Homo sapiens serine and arginine rich splicing factor 7

Homo sapiens stromal antigen 3-like 2

Homo sapiens serine/threonine kinase 25

Homo sapiens testin LIM domain protein

Homo sapiens transcription factor Dp-2

Homo sapiens tropomyosin 1 (alpha)

Homo sapiens TNF receptor associated factor 4

Homo sapiens tripartite motif containing 8

Homo sapiens tetraspanin 33

Homo sapiens UDP-glucose pyrophosphorylase 2

Homo sapiens ubiquinol-cytochrome c reductase, complex III subunit X

Homo sapiens zinc finger DHHC-type containing 3

Homo sapiens zinc finger protein 180

Homo sapiens zinc finger protein 432

Homo sapiens zinc finger RANBP2-type containing 3 